\title{
BLOCK GAUSS AND ANTI-GAUSS QUADRATURE WITH APPLICATION TO NETWORKS*
}

\author{
C. FENU $^{\dagger}$, D. MARTIN ${ }^{\ddagger}$, L. REICHEL ${ }^{\ddagger}$, AND G. RODRIGUEZ ${ }^{\dagger}$
}

\begin{abstract}
Approximations of matrix-valued functions of the form $W^{T} f(A) W$, where $A \in$ $\mathbb{R}^{m \times m}$ is symmetric, $W \in \mathbb{R}^{m \times k}$, with $m$ large and $k \ll m$, has orthonormal columns, and $f$ is a function, can be computed by applying a few steps of the symmetric block Lanczos method to $A$ with initial block-vector $W \in \mathbb{R}^{m \times k}$. Golub and Meurant have shown that the approximants obtained in this manner may be considered block Gauss quadrature rules associated with a matrix-valued measure. This paper generalizes anti-Gauss quadrature rules, introduced by Laurie for real-valued measures, to matrix-valued measures, and shows that under suitable conditions pairs of block Gauss and block anti-Gauss rules provide upper and lower bounds for the entries of the desired matrix-valued function. Extensions to matrix-valued functions of the form $W^{T} f(A) V$, where $A \in \mathbb{R}^{m \times m}$ may be nonsymmetric, and the matrices $V, W \in \mathbb{R}^{m \times k}$ satisfy $V^{T} W=I_{k}$ are also discussed. Approximations of the latter functions are computed by applying a few steps of the nonsymmetric block Lanczos method to $A$ with initial block-vectors $V$ and $W$. We describe applications to the evaluation of functions of a symmetric or nonsymmetric adjacency matrix for a network. Numerical examples illustrate that a combination of block Gauss and anti-Gauss quadrature rules typically provides upper and lower bounds for such problems. We introduce some new quantities that describe properties of nodes in directed or undirected networks, and demonstrate how these and other quantities can be computed inexpensively with the quadrature rules of the present paper.
\end{abstract}

Key words. Gauss quadrature, anti-Gauss quadrature, block Lanczos algorithm, nonsymmetric block Lanczos algorithm, adjacency matrix, network analysis, graph

AMS subject classifications. 65F60, 65D32, 05C82, 91D30

DOI. $10.1137 / 120886261$

1. Introduction. One of the aims of this paper is to discuss the inexpensive computation of bounds or estimates of bounds for expressions of the form

$$
W^{T} f(A) W,
$$

where $A \in \mathbb{R}^{m \times m}$ is a large symmetric matrix, $f$ is a function, which we assume to be analytic in a region in the complex plane that contains the spectrum of $A$, and $W \in \mathbb{R}^{m \times k}$ has orthonormal columns with $1 \leq k \ll m$. We are also interested in the computation of estimates of bounds for more general expressions

$$
W^{T} f(A) V,
$$

where the large matrix $A \in \mathbb{R}^{m \times m}$ may be nonsymmetric and $W, V \in \mathbb{R}^{m \times k}$ satisfy $V^{T} W=I_{k}$. Here and throughout this paper, $I_{k}$ denotes the $k \times k$ identity matrix.

Golub and Meurant $[28,29]$ discuss how the application of a few steps of the symmetric block Lanczos method to a symmetric matrix $A$ with initial block vector $W$

*Received by the editors July 27, 2012; accepted for publication (in revised form) by J. Liesen September 4, 2013; published electronically December 17, 2013. The third author was supported by the Visiting Professor program, University of Cagliari, funded by the Regione Autonoma della Sardegna.

http://www.siam.org/journals/simax/34-4/88626.html

${ }^{\dagger}$ Dipartimento di Matematica e Informatica, Università di Cagliari, 09123 Cagliari, Italy (kate. fenu@unica.it, rodriguez@unica.it). These authors were supported in part by Regione Sardegna grant CRP3_92 and by INdAM-GNCS.

${ }^{\ddagger}$ Department of Mathematical Sciences, Kent State University, Kent, OH 44242 (dmarti49@kent. edu, reichel@math.kent.edu). These authors were supported in part by NSF grant DMS-1115385. 
yields an approximation of (1.1), and show that this approximation can be interpreted as a Gauss-type quadrature rule with respect to a discrete matrix-valued measure. In the special case when the block-size $k$ is one, the symmetric block Lanczos method simplifies to the standard symmetric Lanczos method. Consider this situation, i.e., let $k=1$ and assume that $A$ is symmetric and the function $f$ has derivatives of constant sign in the convex hull of the spectrum of $A$. Then pairs of suitable Gauss, GaussRadau, or Gauss-Lobatto rules yield upper and lower bounds for (1.1). This property follows from the remainder formulas for Gauss-type quadrature rules; see [28, 29] for details. Golub and Meurant $[28,29]$ show that the quadrature rules can be evaluated by using partial Lanczos decompositions of $A$. Applications of this technique to many problems are described in $[3,5,7,14,29,30,31,44]$. Unfortunately, these quadrature rules are not guaranteed to yield upper and lower bounds when pertinent derivatives of $f$ change sign in the convex hull of the spectrum of $A$, and neither are block versions (with block-size $k>1$ ) of the mentioned Gauss-type quadrature rules.

The matrix function (1.2) with a possibly nonsymmetric matrix $A$ can be approximated by a function of a smaller matrix by application of a few steps of the nonsymmetric block Lanczos method with initial block vectors $V$ and $W$. The reduction can be interpreted as a Gauss-type quadrature rule. However, generally this rule is not guaranteed to furnish upper or lower bounds for the elements of (1.2).

Consider the computation of an approximation of the integral $\mathcal{I} f=\int f(t) d \mu(t)$, where $d \mu$ is a positive measure on a real interval such that all moments $\int t^{j} d \mu(t)$, $j=0,1,2, \ldots$, exist. We assume for simplicity that $f$ is real-valued on the support of $d \mu$. Let $\mathcal{G}_{n}$ denote the $n$-point Gauss quadrature rule with respect to $d \mu$. Laurie [37] introduced so-called anti-Gauss quadrature rules for the approximation of $\mathcal{I} f$. The $(n+1)$-point anti-Gauss quadrature rule $\mathcal{H}_{n+1}$ associated with the Gauss rule $\mathcal{G}_{n}$ is characterized by

$$
\left(\mathcal{I}-\mathcal{H}_{n+1}\right) p=-\left(\mathcal{I}-\mathcal{G}_{n}\right) p \quad \forall p \in \mathbb{P}^{2 n+1},
$$

where $\mathbb{P}^{2 n+1}$ denotes the set of all polynomials of degree at most $2 n+1$ (with scalar coefficients). Thus, when $f \in \mathbb{P}^{2 n+1}$, the pair of quadrature rules $\mathcal{G}_{n} f$ and $\mathcal{H}_{n+1} f$ yield upper and lower bounds for $\mathcal{I} f$. In fact, $\mathcal{G}_{n} f=\mathcal{H}_{n+1} f=\mathcal{I} f$ for $f \in \mathbb{P}^{2 n-1}$. For more general functions $f$, the pair of quadrature rules $\mathcal{G}_{n} f$ and $\mathcal{H}_{n+1} f$ provide upper and lower bounds for $\mathcal{I} f$ when the coefficients in an expansion of $f$ in terms of orthonormal polynomials with regard to the measure $d \mu$ decay sufficiently rapidly in magnitude; see [15] and the end of subsection 3.1. This condition is difficult to verify computationally; however, computed examples in [15] show that pairs of Gauss and anti-Gauss quadrature rules indeed yield upper and lower bounds for many integrands that are analytic in a large enough region that contains the interval of integration.

An attraction of the anti-Gauss rule $\mathcal{H}_{n+1}$ is that the property (1.3) is independent of a remainder formula for Gauss quadrature. Pairs of Gauss and anti-Gauss rules can be applied when no useful information with regard to the sign of the quadrature error can be gleaned from a remainder formula. This is the case when the quadrature rule is obtained from the symmetric or nonsymmetric block Lanczos methods with block-size $k>1$. It is one of the aims of this paper to derive block anti-Gauss quadrature rules for the integration of (1.1) and (1.2). The application of pairs of block Gauss and block anti-Gauss rules to (1.1) or (1.2) yields entrywise upper and lower bounds for the desired quantities for suitable functions $f$. Anti-Gauss rules for the approximation of the expressions (1.1) or (1.2) in the special case of block-size $k=1$ are described in [15]. This paper provides an extension to block quadrature. Our derivation builds on work by Golub and Meurant [28, 29] and Duran and Lopez-Rodriguez [18]. 
We apply the block quadrature rules to the analysis of complex networks. Estrada and his collaborators have proposed computable quantities that describe interesting global properties of a complex network; see, e.g., $[6,16,19,21,22,23,24]$. In this application $A$ is a large adjacency matrix for a directed or undirected graph, and $f$ is an analytic function such as the exponential function. The adjacency matrix is symmetric for an undirected network and nonsymmetric for a directed one. The block vectors $W$ and $V$ may, for instance, be chosen to be a few columns of the identity matrix. Other choices will also be discussed.

Most available studies of networks based on analytic functions of adjacency matrices focus on undirected networks; see, e.g., [5, 19, 20, 21, 22]. Discussions on directed networks can be found in $[6,16,23,24]$. The definition of quantities that shed light on properties of networks and the development of efficient numerical methods for their computation are active areas of research. In addition to reviewing several available quantities for studying networks, we introduce a few new ones that can be expressed with the aid of expressions of the forms (1.1) or (1.2), and, therefore, can be approximated by block quadrature rules. We should add that there are many other approaches to network analysis; see, e.g., Bini, Del Corso, and Romani [8], Brezinski and Redivo Zaglia [12], Henson and Sanders [33], and references therein. This paper focuses on methods that require the evaluation of matrix functions that easily can be approximated with the aid of quadrature or block quadrature rules.

While this paper discusses network applications of block quadrature rules, we would like to mention that there also are many other applications of these rules, including solution methods for certain partial differential equations [36] and Tikhonov regularization of large linear discrete ill-posed problems with a matrix right-hand side; see, e.g., [11] for such a problem.

The block quadrature rules discussed in this paper provide efficient and algorithmically simple ways of computing estimates of upper and lower bounds for each element of either (1.1) or (1.2). Section 8 illustrates that when a $k \times k$ submatrix of $f(A)$ is desired, the application of a block method with block-size $k$ once offers an efficient alternative to $O\left(k^{2}\right)$ applications of a standard method with block-size one in terms of the required number of matrix-vector product evaluations. Moreover, block methods are more efficient on computers with a hierarchical memory structure; the evaluation of the product of $A$ with a block-vector $W$ may for modest block-sizes $k$ only be insignificantly slower than the evaluation of the product of $A$ with a single vector. The availability of several processors also can be utilized efficiently; see, e.g., [26] for discussions and examples.

A difficulty when applying the nonsymmetric Lanczos or block Lanczos methods is the occurrence of a particular kind of breakdown, known as serious breakdown. This type of breakdown implies that the computations cannot be continued; see, e.g., $[2,13]$ for discussions on breakdown. For general nonsymmetric matrices $A \in \mathbb{R}^{n \times n}$ and initial vectors $\boldsymbol{v}, \boldsymbol{w} \in \mathbb{R}^{n}$, with many nonvanishing entries, this predicament is unlikely. However, as explained in section 8, serious breakdown is likely to occur when the matrix $A$ is large and sparse and the initial vectors are sparse. This is a common situation when studying large-scale complex networks. Bai, Day, and Ye [2] show that serious breakdown of the nonsymmetric Lanczos and block Lanczos methods can be circumvented by augmenting the original starting vector(s) with a dense vector, thereby increasing the block-size by one. We illustrate this in section 8 .

This paper is organized as follows. Section 2 reviews the connection between graph theory and linear algebra. This connection has been exploited by Estrada and his 
collaborators in their analysis of complex networks. We discuss previously introduced quantities of interest, in addition to presenting several new ones. Section 3 defines the block Gauss and block anti-Gauss quadrature rules that we apply to estimate (1.1) and (1.2), and outlines their computation. In section 4 we discuss the symmetric and nonsymmetric block Lanczos algorithms used to compute the quadrature rules and describe the connection of these algorithms to sequences of orthogonal or biorthogonal matrix polynomials. Section 5 is concerned with the degree of exactness of block Gauss quadrature rules corresponding to the symmetric and nonsymmetric block Lanczos methods, and section 6 describes the computation of block anti-Gauss quadrature rules. A review of how bounds can be computed when the matrix $A$ is symmetric and the block-size is one can be found in section 7. Numerical examples presented in section 8 compare this approach to the application of block Gauss and anti-Gauss rules. The examples focus on network analysis. Section 9 contains concluding remarks.

2. Graph theory and linear algebra. This section reviews some results on the connection between graph theory and linear algebra. A graph $G=\{\mathcal{V}, \mathcal{E}\}$ is defined by a set of vertices $\mathcal{V}$ and a set of edges $\mathcal{E}$. We also will refer to the vertices as nodes. We assume $G$ to be an unweighted graph with $m$ nodes, containing no loops or multiple edges. We consider both undirected graphs, in which travel can occur in both directions along each edge, and directed graphs, in which some or all edges are "one way streets." We assume that $G$ is both large (having a large number, $m$, of nodes) and sparse (having much fewer than $O\left(\mathrm{~m}^{2}\right)$ edges between the nodes). Such graphs arise in numerous scientific and industrial applications, including genetics, epidemiology, energy distribution, and telecommunication; see, e.g., [21]. The adjacency matrix associated with $G$ is the matrix $A=\left[A_{i j}\right] \in \mathbb{R}^{m \times m}$ defined by $A_{i j}=1$ if there is an edge from node $j$ to node $i$, and $A_{i j}=0$ otherwise. The adjacency matrix is symmetric if and only if $G$ is undirected. We will refer to $G$ either as a graph or a network. The notions of walk and path in a graph are important. A walk is a sequence of vertices $v_{1}, v_{2}, \ldots, v_{k}$ such that there is an edge from vertex $v_{i}$ to vertex $v_{i+1}$ for $i=1,2, \ldots, k-1$. Vertices and edges may be repeated. A path is a walk with all vertices distinct.

Given a large graph, it can be useful to extract numerical quantities that describe interesting global properties of the graph, such as the overall importance of a particular node within the network, or the ease of traveling from one node to another. In an undirected network, the degree of a node $i$, which is the number of nodes connected to that node, provides a rough measure of the importance of the node. However, this quantity fails to take into consideration the importance of the nodes connected to node $i$, e.g., how well-connected they are. Analogously, a rough measure of the ease of traveling from node $i$ to node $j$ is the length of the shortest path connecting these nodes. However, this measure fails to take into consideration the possibility that a somewhat longer path may be useful. For example, during rush hour many commuters resort to longer routes to reduce their exposure to traffic. Similarly, the expected time required by a virus to travel from Alice to Bob decreases as the number of their mutual friends increases, and decreases further as the number of friendships between mutual friends increases. Even if Alice and Bob are friends, i.e., a path of length one exists between them, the virus may be transmitted via one or more mutual friends along a path of length larger than one.

The following observation establishes an important link between graph theory and linear algebra, a connection that has been exploited by Estrada and his collaborators in their quest for alternatives to the concepts of degree and shortest path; see, e.g., 
$[19,20,21,22,23,24]$. It is not hard to see that for $\ell \geq 1$, the entry $\left[A^{\ell}\right]_{i j}$ of the matrix $A^{\ell}$ is equal to the number of walks of length $\ell$ starting at node $j$ and ending at node $i$. Thus, given a function

$$
f(A)=\sum_{\ell=0}^{\infty} c_{\ell} A^{\ell}
$$

with nonnegative coefficients $c_{\ell}$ chosen to guarantee convergence, the quantity $[f(A)]_{i j}$ can be interpreted as a measure of the overall ease of traveling from node $j$ to node $i$ within the network. The term $c_{0} I_{m}$ has no specific meaning and is introduced for convenience. We may think of the coefficients $c_{\ell}$ as weights. They are chosen to decrease as $\ell$ increases in order to penalize the contributions of long walks and to secure convergence of the sum (2.1). The choice $c_{\ell}=1 / \ell !$ yields $f(A)=\exp (A)$ and is discussed by Estrada and Higham [21]. Another popular choice are coefficients that yield functions of the form

$$
f(A)=(I-c A)^{-1}
$$

where $c$ is a coefficient small enough so that the representation (2.1) exists; see [10,21].

Estrada and his collaborators have defined the following quantities, relevant to both directed and undirected graphs:

- The $f$-communicability [21] from node $j$ to node $i$, given by $[f(A)]_{i j}$, quantifies the ease of traveling from node $j$ to node $i$.

- The $f$-communicability betweenness [22] of node $r$ is given by

$$
\frac{1}{(m-1)(m-2)} \sum_{i \neq r} \sum_{\substack{j \neq r \\ j \neq i}} \frac{[f(A)]_{i j}-\left[f\left(A_{r}\right)\right]_{i j}}{[f(A)]_{i j}},
$$

where $A_{r}$ is the adjacency matrix of the graph obtained by removing from $G$ all edges involving node $r$. This is a measure of the amount of communication passing through node $r$.

- The average $f$-communicability from node $r$ is defined by

$$
\frac{1}{m-1} \boldsymbol{c}_{r}^{T} f(A) \boldsymbol{e}_{r}
$$

where $\boldsymbol{e}_{r}=[0, \ldots, 0,1,0, \ldots, 0]^{T}$ is the $r$ th axis vector, $\boldsymbol{c}=[1,1, \ldots, 1]^{T}$ is the vector with all entries equal to one, and $\boldsymbol{c}_{r}=\boldsymbol{c}-\boldsymbol{e}_{r}$. This quantity is defined in [20] for $f(A)=\exp (A)$.

The above quantities are applied to symmetric matrices in [20,21,22], but they are of interest for nonsymmetric adjacency matrices, which correspond to directed graphs, as well. In the case of a directed or undirected graph, the $f$-communicability from node $i$ to itself, i.e.,

$$
[f(A)]_{i i}=\boldsymbol{e}_{i}^{T} f(A) \boldsymbol{e}_{i},
$$

is referred to as the $f$-subgraph centrality of node $i$; see [21, 23, 24]. Further quantities relevant for undirected graphs are discussed in [6]. The following quantities also would 
appear to be of interest:

- The $f$-starting convenience of node $i$, given by

$$
m \frac{\boldsymbol{c}^{T} f(A) \boldsymbol{e}_{i}}{\boldsymbol{c}^{T} f(A) \boldsymbol{c}}
$$

quantifies the ease of traveling from node $i$ to anywhere in the network. This is the sum of the communicabilities from node $i$ to all other nodes, scaled so that the average of the quantity over all nodes is one.

- The $f$-ending convenience of node $i$, given by

$$
m \frac{\boldsymbol{e}_{i}^{T} f(A) \boldsymbol{c}}{\boldsymbol{c}^{T} f(A) \boldsymbol{c}}
$$

quantifies the ease of traveling to node $i$ from anywhere in the network. This is the sum of the communicabilities from all other nodes to node $i$, scaled so that the average of the quantity over all nodes is one. The $f$-ending convenience agrees with the $f$-starting convenience when the graph $G$ is undirected.

- The alternative $f$-communicability betweenness of node $r$ is given by

$$
\frac{\boldsymbol{c}_{r}^{T} f(A) \boldsymbol{c}_{r}-\boldsymbol{c}_{r}^{T} f\left(A_{r}\right) \boldsymbol{c}_{r}}{\boldsymbol{c}_{r}^{T} f(A) \boldsymbol{c}_{r}}
$$

This quantity is related to (2.2), but differs from the latter in that it takes into consideration the effect of removing node $r$ on the diagonal elements of $f(A)$, i.e., it takes into account the importance of node $r$ as an intermediate step in closed walks. The scaling is also different from (2.2), as the summation for the latter quantity includes the relative change in each value $[f(A)]_{i j}$ (with $i \neq r, j \neq r, i \neq j$ ) caused by the removal of all edges involving node $r$, whereas all corresponding terms in (2.4) are divided by the same number. A reason for introducing (2.4) is that, different from (2.2), it can be conveniently approximated by (block) Gauss-type quadrature rules. By construction, both quantities (2.2) and (2.4) are between 0 and 1.

We generally suppress the prefix " $f-$ " in the above quantities when the function $f$ is clear from the context.

When $G$ is large, direct evaluation of $f(A)$ generally is not feasible. Benzi and Boito [5] compute bounds for quantities of the form $\boldsymbol{w}^{T} f(A) \boldsymbol{w}$, when $A$ is a symmetric adjacency matrix and $\boldsymbol{w}$ is a vector, by using the connection between the symmetric Lanczos method and Gauss-type quadrature rules described in [28, 29]. In section 8 we instead compute approximations of centralities and communicabilities of nodes by using block Gauss and anti-Gauss quadrature rules evaluated with the symmetric or nonsymmetric block Lanczos methods.

3. Matrices, orthogonal polynomials, and quadrature. This section provides an overview of the techniques based on the use of block Gauss and block antiGauss quadrature rules that we will use to approximate expressions of the form (1.1) and (1.2).

3.1. The symmetric problem $\boldsymbol{W}^{\boldsymbol{T}} \boldsymbol{f}(\boldsymbol{A}) \boldsymbol{W}$. The matrix $A \in \mathbb{R}^{m \times m}$ is assumed to be symmetric throughout this subsection. Anticipating the use of quadrature rules, we first show that the expression (1.1) can be written as a Stieltjes integral. This was first observed by Golub and Meurant [28]. A more recent discussion can be found in [29]. 
Introduce the spectral factorization

$$
A=Q \Lambda Q^{T},
$$

where $Q \in \mathbb{R}^{m \times m}$ is orthogonal and $\Lambda=\operatorname{diag}\left[\lambda_{1}, \ldots, \lambda_{m}\right]$. The eigenvalues are assumed to be ordered according to $\lambda_{1} \leq \cdots \leq \lambda_{m}$. Substituting the spectral factorization (3.1) into (1.1) yields

$$
W^{T} f(A) W=\widetilde{W} f(\Lambda) \widetilde{W}^{T}=\sum_{i=1}^{m} f\left(\lambda_{i}\right) \boldsymbol{\alpha}_{i} \boldsymbol{\alpha}_{i}^{T}=\int f(\lambda) d \alpha(\lambda)=: \mathcal{I} f
$$

where $\widetilde{W}=\left[\boldsymbol{\alpha}_{1}, \ldots, \boldsymbol{\alpha}_{m}\right]=W^{T} Q \in \mathbb{R}^{k \times m}$ and $\alpha: \mathbb{R} \rightarrow \mathbb{R}^{k \times k}$ is a discrete matrixvalued distribution with jumps $\boldsymbol{\alpha}_{i} \boldsymbol{\alpha}_{i}^{T}$ at the eigenvalues $\lambda_{i}$ of $A$.

Following $[28,29]$, we show in section 4 that there is a sequence of polynomials $p_{j}$ that are orthonormal with respect to a bilinear form defined by $d \alpha$ and have $k \times k$ matrix coefficients. The polynomials satisfy a three-term recursion relation of the form

$$
\begin{aligned}
\lambda p_{j-1}(\lambda) & =p_{j}(\lambda) \Gamma_{j}+p_{j-1}(\lambda) \Omega_{j}+p_{j-2}(\lambda) \Gamma_{j-1}^{T}, \quad j=1,2, \ldots, \\
p_{0}(\lambda) & :=I_{k}, \quad p_{-1}(\lambda):=O_{k},
\end{aligned}
$$

where $O_{k}$ denotes the $k \times k$ zero matrix. For each $j$, the recursion coefficients $\Gamma_{j}$ and $\Omega_{j}$ are $k \times k$ matrices with real entries. Moreover, $\Omega_{j}$ is symmetric and $\Gamma_{j}$ can be chosen to be upper triangular; see section 4 . The $p_{j}$ are orthonormal with respect to a matrix-valued bilinear form defined by the measure $d \alpha$ in (3.2); see Theorem 1 below. Defining

$$
P_{N}(\lambda):=\left[p_{0}(\lambda), \ldots, p_{N-1}(\lambda)\right] \in \mathbb{R}^{k \times k N},
$$

it follows that

$$
\lambda P_{N}(\lambda)=P_{N}(\lambda) J_{N}+p_{N}(\lambda) \Gamma_{N} E_{N}^{T},
$$

where

$$
J_{N}:=\left[\begin{array}{ccccc}
\Omega_{1} & \Gamma_{1}^{T} & & & \\
\Gamma_{1} & \Omega_{2} & \Gamma_{2}^{T} & & \\
& \ddots & \ddots & \ddots & \\
& & \Gamma_{N-2} & \Omega_{N-1} & \Gamma_{N-1}^{T} \\
& & & \Gamma_{N-1} & \Omega_{N}
\end{array}\right] \in \mathbb{R}^{k N \times k N}
$$

Throughout this section, $E_{i}:=\left[\boldsymbol{e}_{(i-1) k+1}, \ldots, \boldsymbol{e}_{i k}\right]$ denotes a "block axis vector" of appropriate size with $k \times k$ blocks. Thus, the $i$ th block of $E_{i}$ is $I_{k}$ and all other blocks vanish. The matrix $J_{N}$ is symmetric, block-tridiagonal, and has bandwidth $2 k+1$. It is determined by $N$ steps of the symmetric block Lanczos method with block-size $k$ described in section 4 . We remark that the polynomials $p_{j}$ are considered for theoretical purposes only; they are not explicitly computed.

Introduce the spectral factorization $J_{N}=Y_{N} \Theta_{N} Y_{N}^{T}$, where

$$
Y_{N}=\left[\boldsymbol{y}_{1}^{(N)}, \ldots, \boldsymbol{y}_{k N}^{(N)}\right] \in \mathbb{R}^{k N \times k N}, \quad \Theta_{N}=\operatorname{diag}\left[\theta_{1}^{(N)}, \ldots, \theta_{k N}^{(N)}\right] \in \mathbb{R}^{k N \times k N},
$$


where the matrix $Y_{N}$ is orthogonal and the eigenvalues are ordered according to $\theta_{1}^{(N)} \leq \cdots \leq \theta_{k N}^{(N)}$. Consider the expression

$$
\mathcal{G}_{N} f:=\sum_{i=1}^{k N} f\left(\theta_{i}^{(N)}\right) \boldsymbol{u}_{i}^{(N)}\left(\boldsymbol{u}_{i}^{(N)}\right)^{T}
$$

where each vector $\boldsymbol{u}_{i}^{(N)} \in \mathbb{R}^{k}$ consists of the first $k$ elements of $\boldsymbol{y}_{i}^{(N)}$. It is shown in $[28,29]$ that $\mathcal{G}_{N}$ is a Gauss quadrature rule with respect to a matrix-valued bilinear form defined by the measure $d \alpha$ in (3.2), i.e.,

$$
\mathcal{G}_{N} f=\mathcal{I} f \quad \forall f \in \mathbb{P}^{2 N-1}
$$

related results are discussed in [45]. An alternative and more concise proof of this result is provided in section 5 . We refer to $\mathcal{G}_{N}$ as an $N$-block Gauss quadrature rule associated with a bilinear form determined by the matrix measure $d \alpha$. This quadrature rule allows the matrix representation

$$
\begin{aligned}
\mathcal{G}_{N} f & =\sum_{i=1}^{k N} f\left(\theta_{i}^{(N)}\right) \boldsymbol{u}_{i}^{(N)}\left(\boldsymbol{u}_{i}^{(N)}\right)^{T}=\left[\boldsymbol{u}_{1}^{(N)}, \ldots, \boldsymbol{u}_{k N}^{(N)}\right] f\left(\Theta_{N}\right)\left[\boldsymbol{u}_{1}^{(N)}, \ldots, \boldsymbol{u}_{k N}^{(N)}\right]^{T} \\
& =E_{1}^{T} Y_{N} f\left(\Theta_{N}\right) Y_{N}^{T} E_{1}=E_{1}^{T} f\left(J_{N}\right) E_{1},
\end{aligned}
$$

which shows that for certain functions $f$, such as $f(x)=\exp (x)$ and $f(x)=1 /(1-c x)$, where $c$ is a suitable constant, the block Gauss rule $\mathcal{G}_{N}$ can be evaluated efficiently via the right-hand side of (3.7) without computing the spectral factorization of $J_{N}$. For instance, when $f(x)=\exp (x)$, we can compute $f\left(J_{N}\right)$ by using a Padé approximant; see Higham [34] for discussions on methods for the evaluation of many matrix functions for matrices of small to moderate size.

We turn to the derivation of block anti-Gauss rules. Proceeding similarly as Laurie [37] for the case of a real-valued positive measure (cf. (1.3)) we define the $(N+1)$-block anti-Gauss quadrature rule $\mathcal{H}_{N+1}$ to be an $(N+1)$-block quadrature rule such that

$$
\left(\mathcal{I}-\mathcal{H}_{N+1}\right) f=-\left(\mathcal{I}-\mathcal{G}_{N}\right) f, \quad f \in \mathbb{P}^{2 N+1} .
$$

Since (3.8) implies that

$$
\mathcal{H}_{N+1} f=\left(2 \mathcal{I}-\mathcal{G}_{N}\right) f, \quad f \in \mathbb{P}^{2 N+1},
$$

it follows that $\mathcal{H}_{N+1}$ is the (ordinary) $(N+1)$-block Gauss quadrature rule with respect to the bilinear form determined by the matrix-valued function $2 \mathcal{I}-\mathcal{G}_{N}$. We note that the average rule

$$
\mathcal{A}_{N+1}:=\frac{1}{2}\left(\mathcal{H}_{N+1}+\mathcal{G}_{N}\right)
$$

is exact for all polynomials of degree up to and including $2 N+1$. 
Similarly as above, there is a sequence of orthonormal polynomials $\tilde{p}_{j}$, with $k \times k$ matrix coefficients, such that

$$
\begin{aligned}
\lambda \tilde{p}_{j-1}(\lambda) & =\tilde{p}_{j}(\lambda) \tilde{\Gamma}_{j}+\tilde{p}_{j-1}(\lambda) \tilde{\Omega}_{j}+\tilde{p}_{j-2}(\lambda) \tilde{\Gamma}_{j-1}^{T}, \quad j=1,2, \ldots, \\
\tilde{p}_{0}(\lambda) & :=I_{k}, \quad \tilde{p}_{-1}(\lambda):=O_{k},
\end{aligned}
$$

where the orthonormality is, with respect to a bilinear form, defined by the matrixvalued measure induced by the function $2 \mathcal{I}-\mathcal{G}_{N}$.

We show in section 6 how to determine the symmetric block tridiagonal matrix

$$
\tilde{J}_{N+1}=\left[\begin{array}{ccccc}
\tilde{\Omega}_{1} & \tilde{\Gamma}_{1}^{T} & & & \\
\tilde{\Gamma}_{1} & \tilde{\Omega}_{2} & \tilde{\Gamma}_{2}^{T} & & \\
& \ddots & \ddots & \ddots & \\
& & \tilde{\Gamma}_{N-1} & \tilde{\Omega}_{N} & \tilde{\Gamma}_{N}^{T} \\
& & & \tilde{\Gamma}_{N} & \tilde{\Omega}_{N+1}
\end{array}\right] \in \mathbb{R}^{k(N+1) \times k(N+1)}
$$

associated with the anti-Gauss rule $\mathcal{H}_{N+1}$ with almost no work from the matrix $J_{N+1}$ related to the $(N+1)$-block Gauss quadrature rule $\mathcal{G}_{N+1}$ defined by a bilinear form determined by the matrix measure $d \alpha$. Analogously to $(3.7)$, the $(N+1)$-block antiGauss quadrature rule (3.9) allows the matrix representation

$$
\mathcal{H}_{N+1} f=E_{1}^{T} f\left(\tilde{J}_{N+1}\right) E_{1} .
$$

Our application to network analysis only requires the evaluation of (1.1) for functions that can be represented by a series with scalar coefficients. However, it will be convenient to extend $\mathcal{I}$ and $\mathcal{G}_{N}$ to allow matrix-valued functions $f$ and $g$ that can be represented by a series with $k \times k$ matrix coefficients with real entries. For later convenience we define the quantities

$$
\begin{aligned}
\mathcal{I}(f, g) & :=\sum_{i=1}^{m} f^{T}\left(\lambda_{i}\right) \boldsymbol{\alpha}_{i} \boldsymbol{\alpha}_{i}^{T} g\left(\lambda_{i}\right) \\
\mathcal{G}_{N}(f, g) & :=\sum_{i=1}^{k N} f^{T}\left(\theta_{i}^{(N)}\right) \boldsymbol{u}_{i}^{(N)}\left(\boldsymbol{u}_{i}^{(N)}\right)^{T} g\left(\theta_{i}^{(N)}\right),
\end{aligned}
$$

which will be used to show that, indeed, the mentioned properties of the anti-Gauss and average quadrature rules hold.

We conclude this section with some comments on why pairs of $N$-block Gauss rules (3.6) and $(N+1)$-block anti-Gauss rules (3.13) may provide elementwise upper and lower bounds for (3.2) when the integrand is analytic in a sufficiently large region in the complex plane that contains the support of the measure $d \alpha$. Assume for notational simplicity that there are infinitely many orthogonal polynomials (3.3) and that $f$ admits a representation of the form

$$
f(x)=\sum_{i=0}^{\infty} C_{i} p_{i}(x)
$$


where the coefficients $C_{i}$ are $k \times k$ matrices. Assuming that $\mathcal{I}(f, f)$ is finite, one can show that the matrices $C_{i}$ must converge to zero as $i$ increases. Moreover, we have

$$
\begin{aligned}
\mathcal{I} f & =C_{0}, \\
\mathcal{G}_{N} f & =C_{0}+\sum_{i=2 N}^{\infty} C_{i} \mathcal{G}_{N} p_{i}=C_{0}+C_{2 N} \mathcal{G}_{N} p_{2 N}+C_{2 N+1} \mathcal{G}_{N} p_{2 N+1}+\sum_{i=2 N+2}^{\infty} C_{i} \mathcal{G}_{N} p_{i}, \\
\mathcal{H}_{N+1} f & =C_{0}+\sum_{i=2 N}^{\infty} C_{i} \mathcal{H}_{N+1} p_{i} \\
& =C_{0}+C_{2 N} \mathcal{H}_{N+1} p_{2 N}+C_{2 N+1} \mathcal{H}_{N+1} p_{2 N+1}+\sum_{i=2 N+2}^{\infty} C_{i} \mathcal{H}_{N+1} p_{i} \\
& =C_{0}-C_{2 N} \mathcal{G}_{N} p_{2 N}-C_{2 N+1} \mathcal{G}_{N} p_{2 N+1}+\sum_{i=2 N+2}^{\infty} C_{i} \mathcal{H}_{N+1} p_{i},
\end{aligned}
$$

where we have used (3.9). Now, if the coefficient matrices $C_{i}$ decay in norm sufficiently rapidly with increasing $i$, then the approximations

$$
\begin{aligned}
\mathcal{G}_{N} f-\mathcal{I} f & \approx C_{2 N} \mathcal{G}_{N} p_{2 N}+C_{2 N+1} \mathcal{G}_{N} p_{2 N+1}, \\
\mathcal{H}_{N+1} f-\mathcal{I} f & \approx-C_{2 N} \mathcal{G}_{N} p_{2 N}-C_{2 N+1} \mathcal{G}_{N} p_{2 N+1}
\end{aligned}
$$

suggest that the componentwise errors of the quadrature rules $\mathcal{G}_{N} f$ and $\mathcal{H}_{N+1} f$ are roughly equal in magnitude and of opposite sign. The norm of the matrices $C_{i}$ decays quickly to zero when $i$ increases if $f$ is analytic in a large simply connected region in the complex plane that contains the support of the measure $d \alpha$ and has its boundary far away from the support.

3.2. The nonsymmetric problem $\boldsymbol{W}^{\boldsymbol{T}} \boldsymbol{f}(\boldsymbol{A}) \boldsymbol{V}$. We assume that $A \in \mathbb{R}^{m \times m}$ is diagonalizable and introduce the spectral factorization $A=Q \Lambda Q^{-1}$, where $Q \in$ $\mathbb{C}^{m \times m}$ is nonsingular and $\Lambda=\operatorname{diag}\left[\lambda_{1}, \ldots, \lambda_{m}\right]$. When $A \neq A^{T}$, the eigenvalues $\lambda_{i}$ may be complex-valued. Letting

$$
\widetilde{W}=\left[\boldsymbol{\alpha}_{1}, \ldots, \boldsymbol{\alpha}_{m}\right]=W^{T} Q \in \mathbb{C}^{k \times m}, \quad \widetilde{V}=\left[\boldsymbol{\beta}_{1}, \ldots, \boldsymbol{\beta}_{m}\right]=\left(Q^{-1} V\right)^{H} \in \mathbb{C}^{k \times m},
$$

we obtain

$$
W^{T} f(A) V=\widetilde{W} f(\Lambda) \widetilde{V}^{H}=\sum_{i=1}^{m} f\left(\lambda_{i}\right) \boldsymbol{\alpha}_{i} \boldsymbol{\beta}_{i}^{H}=: \mathcal{I} f
$$

where the superscript ${ }^{H}$ denotes transposition and complex conjugation.

We will show in section 4 that there are two sequences of polynomials $p_{j}$ and $q_{j}, j=0,1, \ldots$, with $k \times k$ matrix coefficients, that are biorthogonal with respect to a bilinear form determined by the matrix-valued function $\mathcal{I}$ in (3.16) and satisfy recursion relations of the form

$$
\begin{aligned}
\lambda p_{j-1}(\lambda) & =p_{j}(\lambda) \Gamma_{j}+p_{j-1}(\lambda) \Omega_{j}+p_{j-2}(\lambda) \Delta_{j-1}^{T}, \\
\lambda q_{j-1}(\lambda) & =q_{j}(\lambda) \Delta_{j}+q_{j-1}(\lambda) \Omega_{j}^{T}+q_{j-2}(\lambda) \Gamma_{j-1}^{T}, \\
p_{0}(\lambda) & :=I_{k}, \quad q_{0}(\lambda):=I_{k}, \quad p_{-1}(\lambda):=O_{k}, \quad q_{-1}(\lambda):=O_{k},
\end{aligned}
$$


for $j=1,2, \ldots$ The matrix recursion coefficients $\Gamma_{j}, \Omega_{j}$, and $\Delta_{j}$ are real $k \times k$ matrices. Letting

$$
\begin{aligned}
P_{N}(\lambda) & :=\left[p_{0}(\lambda), \ldots, p_{N-1}(\lambda)\right] \in \mathbb{R}^{k \times k N}, \\
Q_{N}(\lambda) & :=\left[q_{0}(\lambda), \ldots, q_{N-1}(\lambda)\right] \in \mathbb{R}^{k \times k N},
\end{aligned}
$$

the recursion relations (3.17) can be expressed as

$$
\begin{aligned}
\lambda P_{N}(\lambda) & =P_{N}(\lambda) J_{N}+p_{N}(\lambda) \Gamma_{N} E_{N}^{T}, \\
\lambda Q_{N}(\lambda) & =Q_{N}(\lambda) J_{N}^{T}+q_{N}(\lambda) \Delta_{N} E_{N}^{T},
\end{aligned}
$$

where

$$
J_{N}:=\left[\begin{array}{ccccc}
\Omega_{1} & \Delta_{1}^{T} & & & \\
\Gamma_{1} & \Omega_{2} & \Delta_{2}^{T} & & \\
& \ddots & \ddots & \ddots & \\
& & \Gamma_{N-2} & \Omega_{N-1} & \Delta_{N-1}^{T} \\
& & & \Gamma_{N-1} & \Omega_{N}
\end{array}\right] \in \mathbb{R}^{k N \times k N}
$$

is a block-tridiagonal matrix determined by $N$ steps of the nonsymmetric block Lanczos method described in [2] and discussed in section 4 . We remark that the polynomials $p_{j}$ and $q_{j}$ are considered for theoretical purposes only; they are never explicitly stored or utilized in the computations.

We assume that $J_{N}$ is diagonalizable and write $J_{N}=Y_{N} \Theta_{N} Y_{N}^{-1}$, where

$$
Y_{N}=\left[\boldsymbol{y}_{1}^{(N)}, \ldots, \boldsymbol{y}_{k N}^{(N)}\right] \in \mathbb{C}^{k N \times k N}, \quad \Theta_{N}=\operatorname{diag}\left[\theta_{1}^{(N)}, \ldots, \theta_{k N}^{(N)}\right] \in \mathbb{C}^{k N \times k N}
$$

Letting $Z_{N}:=\left[\boldsymbol{z}_{1}^{(N)}, \ldots, \boldsymbol{z}_{k N}^{(N)}\right]=Y_{N}^{-H}$, we obtain

$$
J_{N}=Y_{N} \Theta_{N} Z_{N}^{H}, \quad J_{N}^{T} Z_{N}=Z_{N} \bar{\Theta}_{N}
$$

where the bar denotes complex conjugation. Since the matrices $A, V, W$ have real entries only, so does $J_{N}$ and, therefore, $J^{T}=J^{H}$.

Consider the quadrature rule

$$
\mathcal{G}_{N} f:=\sum_{i=1}^{k N} f\left(\theta_{i}^{(N)}\right) \boldsymbol{u}_{i}^{(N)}\left(\boldsymbol{v}_{i}^{(N)}\right)^{H}
$$

with respect to a bilinear form determined by $\mathcal{I}$ defined by (3.16). Each vector $\boldsymbol{u}_{i}^{(N)} \in \mathbb{C}^{k}$ consists of the first $k$ elements of the (right) eigenvector $\boldsymbol{y}_{i}^{(N)}$ of $J_{N}$, and each vector $\boldsymbol{v}_{i}^{(N)} \in \mathbb{C}^{k}$ is made up of the first $k$ elements of the (right) eigenvector $\boldsymbol{z}_{i}^{(N)}$ of $J_{N}^{T}$. We will show in section 5 that

$$
\mathcal{G}_{N} f=\mathcal{I} f \quad \forall f \in \mathbb{P}^{2 N-1} .
$$

For this reason, we refer to $\mathcal{G}_{N}$ as an $N$-block nonsymmetric Gauss quadrature rule associated with $\mathcal{I}$. As for the symmetric case considered in subsection 3.1, this quadrature rule can be expressed as

$$
\mathcal{G}_{N} f=E_{1}^{T} f\left(J_{N}\right) E_{1},
$$


where the matrix $J_{N}$ is given by (3.20). In our applications, the matrix $J_{N}$ is small enough to allow the evaluation of the function $f\left(J_{N}\right)$ by methods designed for small to medium-sized matrices; see [34] for such methods.

Similarly as in subsection 3.1, we seek to determine a matrix-valued $(N+1)$-block anti-Gauss quadrature rule $\mathcal{H}_{N+1}$ such that

$$
\left(\mathcal{I}-\mathcal{H}_{N+1}\right) f=-\left(\mathcal{I}-\mathcal{G}_{N}\right) f, \quad f \in \mathbb{P}^{2 N+1} .
$$

This relation implies, analogously to the discussion following (3.8), that $\mathcal{H}_{N+1}$ is an $(N+1)$-block Gauss quadrature rule with respect to a bilinear form determined by the matrix-valued function $2 \mathcal{I}-\mathcal{G}_{N}$. The average rule $(3.10)$ with $\mathcal{G}_{N}$ and $\mathcal{H}_{N+1}$ defined by (3.21) and (3.23), respectively, is exact for all $p \in \mathbb{P}^{2 N+1}$.

Analogously to the discussion above, there are sequences of polynomials $\tilde{p}_{j}$ and $\tilde{q}_{j}, j=0,1, \ldots$, with real $k \times k$ matrix coefficients, that are biorthogonal with respect to a bilinear form determined by the matrix-valued function $2 \mathcal{I}-\mathcal{G}_{N}$ and satisfy recursion relations of the form

$$
\begin{aligned}
\lambda \tilde{p}_{j-1}(\lambda) & =\tilde{p}_{j}(\lambda) \tilde{\Gamma}_{j}+\tilde{p}_{j-1}(\lambda) \tilde{\Omega}_{j}+\tilde{p}_{j-2}(\lambda) \tilde{\Delta}_{j-1}^{T}, \\
\lambda \tilde{q}_{j-1}(\lambda) & =\tilde{q}_{j}(\lambda) \tilde{\Delta}_{j}+\tilde{q}_{j-1}(\lambda) \tilde{\Omega}_{j}^{T}+\tilde{q}_{j-2}(\lambda) \tilde{\Gamma}_{j-1}^{T}, \\
\tilde{p}_{0}(\lambda) & :=I_{k}, \quad \tilde{q}_{0}(\lambda):=I_{k}, \quad \tilde{p}_{-1}(\lambda):=O_{k}, \quad \tilde{q}_{-1}(\lambda):=O_{k},
\end{aligned}
$$

for $j=1,2, \ldots$.

We will show in section 6 how to determine the associated matrix of matrix recursion coefficients

$$
\tilde{J}_{N+1}=\left[\begin{array}{ccccc}
\tilde{\Omega}_{1} & \tilde{\Delta}_{1}^{T} & & & \\
\tilde{\Gamma}_{1} & \tilde{\Omega}_{2} & \tilde{\Delta}_{2}^{T} & & \\
& \ddots & \ddots & \ddots & \\
& & \tilde{\Gamma}_{N-1} & \tilde{\Omega}_{N} & \tilde{\Delta}_{N}^{T} \\
& & & \tilde{\Gamma}_{N} & \tilde{\Omega}_{N+1}
\end{array}\right] \in \mathbb{R}^{k(N+1) \times k(N+1)}
$$

with almost no work, from the matrix (3.20) with $N$ replaced by $N+1$. The $(N+1)$ block nonsymmetric anti-Gauss rule allows the matrix representation

$$
\mathcal{H}_{N+1} f=E_{1}^{T} f\left(\tilde{J}_{N+1}\right) E_{1},
$$

analogous to (3.13).

Similarly as at the end of subsection 3.1 , we extend $\mathcal{I}$ and $\mathcal{G}_{N}$ to allow matrixvalued functions $f$ and $g$ that can be represented by a series with $k \times k$ matrix coefficients. Thus, we define

$$
\begin{aligned}
\mathcal{I}(f, g) & :=\sum_{i=1}^{m} f^{H}\left(\bar{\lambda}_{i}\right) \boldsymbol{\alpha}_{i} \boldsymbol{\beta}_{i}^{H} g\left(\lambda_{i}\right) \\
\mathcal{G}_{N}(f, g) & :=\sum_{i=1}^{k N} f^{H}\left(\bar{\theta}_{i}^{(N)}\right) \boldsymbol{u}_{i}^{(N)}\left(\boldsymbol{v}_{i}^{(N)}\right)^{H} g\left(\theta_{i}^{(N)}\right) .
\end{aligned}
$$

An argument similar to the one at the end of subsection 3.1 can be made regarding the computation of bounds for (3.16) via (3.21) and (3.26). Thus, for integrands for which an expansion in terms of biorthogonal polynomials converges quickly, pairs of $N$-block Gauss and $(N+1)$-block anti-Gauss quadrature rules typically provide entrywise upper and lower bounds. 
4. Block Lanczos methods. In this section we describe the symmetric and nonsymmetric block Lanczos methods, which are used to compute the block Gausstype quadrature rules introduced in the previous section.

4.1. The symmetric block Lanczos method. This subsection follows the development in $[28,29]$. Let the matrix $X_{1} \in \mathbb{R}^{m \times k}$ have orthonormal columns. In our application to approximating the expression (1.1), we let $X_{1}=W$. Define $X_{0}:=O \in \mathbb{R}^{m \times k}$. The recursion relations of the symmetric block Lanczos method are given by

$$
\begin{aligned}
\Omega_{j} & =X_{j}^{T} A X_{j}, \\
R_{j} & =A X_{j}-X_{j} \Omega_{j}-X_{j-1} \Gamma_{j-1}^{T}, \quad j=1, \ldots, N, \\
X_{j+1} \Gamma_{j} & =R_{j},
\end{aligned}
$$

and can be expressed in the form

$$
A\left[X_{1}, \ldots, X_{N}\right]=\left[X_{1}, \ldots, X_{N}\right] J_{N}+X_{N+1} \Gamma_{N} E_{N}^{T} .
$$

Here $X_{j+1} \Gamma_{j}=R_{j}$ is a QR factorization such that $X_{j+1} \in \mathbb{R}^{m \times k}$ has orthonormal columns and $\Gamma_{j} \in \mathbb{R}^{k \times k}$ is upper triangular. The block tridiagonal matrix $J_{N}$ is the same as in (3.5). The symmetric block Lanczos method is said to break down at the $j$ th step if $R_{j}$ is (numerically) rank deficient. In this case, the computations can be continued by replacing (numerically) linearly dependent columns of $X_{j+1}$ by arbitrary columns that are orthogonal to the ranges of the matrices $R_{j}$ and $X_{1}, \ldots, X_{j}$, and then computing the QR factorization $X_{j+1} \Gamma_{j}=R_{j}$. The upper triangular matrix $\Gamma_{j} \in \mathbb{R}^{k \times k}$ so obtained is necessarily singular. For ease of exposition, we assume that the block Lanczos method does not break down during the first $N$ steps. These steps are required to compute the quadrature rules $\mathcal{G}_{N}$ and $\mathcal{H}_{N+1}$. We remark that step $N+1$ of the recursions (4.1) determines the matrices $\Omega_{N+1}, R_{N+1}, X_{N+2}$, and $\Gamma_{N+1}$. However, of these matrices, we need only $\Omega_{N+1}$ to determine $\mathcal{H}_{N+1}$. Therefore, a breakdown in step $N+1$ does not affect the computation of $\mathcal{H}_{N+1}$.

Assuming that no breakdown occurs in the first $N$ steps of the recursions (4.1), each upper triangular matrix $\Gamma_{j}$, for $1 \leq j \leq N$, is invertible. These matrices may be chosen to have positive diagonal elements.

By construction, the block-vectors $X_{i} \in \mathbb{R}^{m \times k}$ satisfy

$$
X_{i}^{T} X_{j}=\delta_{i j} I_{k},
$$

where $\delta_{i j}$ is the Kronecker $\delta$-function, i.e., $\delta_{i i}=1$ for all $i$ and $\delta_{i j}=0$ for $i \neq j$.

One can show by induction that

$$
X_{i+1}=\sum_{j=0}^{i} A^{j} X_{1} C_{j}^{(i)}, \quad 0 \leq i \leq N,
$$

for suitable matrices $C_{j}^{(i)} \in \mathbb{R}^{k \times k}$. Similarly as Golub and Meurant [28, 29], we consider the sequence of matrix polynomials $p_{i}$ defined by

$$
p_{i}(\lambda)=\sum_{j=0}^{i} \lambda^{j} C_{j}^{(i)}, \quad 0 \leq i \leq N .
$$


The following result from $[28,29]$ shows that these polynomials are orthonormal with respect to the bilinear form (3.14).

Theorem 1. Let $\mathcal{I}$ be defined by (3.14). Then the polynomials (4.3) satisfy

$$
\mathcal{I}\left(p_{i}, p_{j}\right)=X_{i+1}^{T} X_{j+1}=\delta_{i j} I_{k}, \quad 0 \leq i, j \leq N .
$$

Proof. This result is shown in $[28,29]$. A proof of a generalization to the nonsymmetric setting is provided in subsection 4.2 below; see Theorem 3 .

One can show that, under the assumptions following (4.2), the matrix polynomials satisfy the recursion relation (3.3), which is analogous to (4.1).

We now state an important characterization of the eigenvalues and eigenvectors of the matrix $J_{N}$ in (3.5) and (4.2). This result is part of [18, Theorem 1.1]. It is also reported in $[28,29]$.

TheOrem 2. The eigenvalues of $J_{N}$ are the zeros of $\operatorname{det}\left[p_{N}(\lambda)\right]$. Furthermore, defining $P_{N}$ by (3.4), the unit (right) eigenvector $\boldsymbol{y}_{r}^{(N)}$ of $J_{N}$ corresponding to the eigenvalue $\theta_{r}^{(N)}$ is given by $\boldsymbol{y}_{r}^{(N)}=P_{N}^{T}\left(\theta_{r}^{(N)}\right) \boldsymbol{u}_{r}^{(N)}$, where $\boldsymbol{u}_{r}^{(N)}$ consists of the first $k$ components of $\boldsymbol{y}_{r}^{(N)}$. Moreover, $p_{N}^{T}\left(\theta_{r}^{(N)}\right) \boldsymbol{u}_{r}^{(N)}=\mathbf{0}$.

Proof. A proof of this result can be found in [18]. Theorem 4 below and its proof provide a generalization to the nonsymmetric setting.

4.2. The nonsymmetric block Lanczos method. Assume that the matrices $V_{1}, W_{1} \in \mathbb{R}^{m \times k}$ satisfy $V_{1}^{T} W_{1}=I_{k}$, and let $V_{0} \Delta_{0}^{T}, W_{0} \Gamma_{0}^{T} \in \mathbb{R}^{m \times k}$ be zero-matrices. When seeking to approximate the expression (1.2), we let $V_{1}=V$ and $W_{1}=W$. The following recursion relations described by Bai, Day, and Ye [2] determine the first $N$ steps of the nonsymmetric block Lanczos method:

$$
\begin{aligned}
\Omega_{j} & =W_{j}^{T}\left(A V_{j}-V_{j-1} \Delta_{j-1}^{T}\right), \\
R_{j} & =A V_{j}-V_{j} \Omega_{j}-V_{j-1} \Delta_{j-1}^{T}, \\
S_{j} & =A^{T} W_{j}-W_{j} \Omega_{j}^{T}-W_{j-1} \Gamma_{j-1}^{T}, \\
Q_{R} R_{R} & =R_{j}, \quad Q_{S} R_{S}=S_{j}, \\
W \Sigma V^{T} & =Q_{S}^{T} Q_{R}, \\
V_{j+1} & =Q_{R} V \Sigma^{-1 / 2}, \quad W_{j+1}=Q_{S} W \Sigma^{-1 / 2}, \ldots, N, \\
\Gamma_{j} & =\Sigma^{1 / 2} V^{T} R_{R}, \quad \Delta_{j}=\Sigma^{1 / 2} W^{T} R_{S} .
\end{aligned}
$$

Here $Q_{R} R_{R}=R_{j}$ and $Q_{S} R_{S}=S_{j}$ are QR factorizations, where $Q_{R}, Q_{S} \in \mathbb{R}^{m \times k}$ have orthonormal columns and $R_{R}, R_{S} \in \mathbb{R}^{k \times k}$ are upper triangular. The factorization $W \Sigma V^{T}=Q_{S}^{T} Q_{R}$ is a singular value decomposition of the right-hand side matrix. The recursions (4.4) can be summarized as

$$
\begin{aligned}
A\left[V_{1}, \ldots, V_{N}\right] & =\left[V_{1}, \ldots, V_{N}\right] J_{N}+V_{N+1} \Gamma_{N} E_{N}^{T}, \\
A^{T}\left[W_{1}, \ldots, W_{N}\right] & =\left[W_{1}, \ldots, W_{N}\right] J_{N}^{T}+W_{N+1} \Delta_{N} E_{N}^{T},
\end{aligned}
$$

where $J_{N}$ is the matrix (3.20).

The recursion formulas (4.4) provide one of many possible implementations of the nonsymmetric block Lanczos method; see [2] for a discussion on the advantages of this particular implementation. We say that the nonsymmetric block Lanczos method breaks down at step $j$ if $S_{j}^{T} R_{j}$ is (numerically) singular. The problem of breakdown is more complicated for the nonsymmetric block Lanczos method than for 
its symmetric counterpart. While breakdown of the symmetric block Lanczos method can always be remedied by the introduction of one or several new vectors, this is not the case for the nonsymmetric block Lanczos method. Instead, the recursions may have to be terminated. This situation is referred to as serious breakdown. A sufficient condition for serious breakdown at step $j$ is that the matrix $S_{j}^{T} R_{j}$ is singular, and both matrices $S_{j}$ and $R_{j}$ are of full rank. Bai, Day, and Ye [2] provide a thorough discussion on breakdown of the recursions (4.4) and show that serious breakdown can be circumvented by restarting the nonsymmetric block Lanczos method after introducing an appropriate additional vector in the initial block-vectors $W_{1}$ and $V_{1}$ (increasing the block-size by 1). As already mentioned, breakdown is an important practical concern when applying the nonsymmetric Lanczos method to the problem (1.2) with sparse $A, W$, and $V$.

When no breakdown occurs during the recursions (4.4), the matrices $\Gamma_{j}$ and $\Delta_{j}$ are nonsingular for $1 \leq j \leq N$. We may assume that the initial block-vectors $W_{1}$ and $V_{1}$ have been suitably augmented to avoid breakdown. We illustrate augmentation in section 8 .

By construction, the block-vectors $W_{i}$ and $V_{i}$ are biorthogonal, i.e., they satisfy

$$
V_{i}^{T} W_{j}=\delta_{i j} I_{k}
$$

One can show by induction that

$$
\begin{aligned}
V_{i+1} & =\sum_{j=0}^{i} A^{j} V_{1} C_{j}^{(i)}, \\
W_{i+1} & =\sum_{j=0}^{i}\left(A^{T}\right)^{j} W_{1} D_{j}^{(i)},
\end{aligned}
$$

for suitably chosen matrices $C_{j}^{(i)}, D_{j}^{(i)} \in \mathbb{R}^{k \times k}$.

Define the matrix polynomials $p_{i}$ and $q_{i}$ by

$$
\begin{aligned}
p_{i}(\lambda) & :=\sum_{j=0}^{i} \lambda^{j} C_{j}^{(i)}, \\
q_{i}(\lambda):=\sum_{j=0}^{i} \lambda^{j} D_{j}^{(i)}, &
\end{aligned}
$$

We now show that these polynomials are biorthogonal with respect to the bilinear form (3.27). This is a generalization of [28, Theorem 4.4] and of Theorem 1 from the previous subsection.

Theorem 3. Let $\mathcal{I}$ be defined by (3.27) and let $V_{1}=V$ and $W_{1}=W$, where $W$ and $V$ are the block vectors in (1.2). Then the polynomials (4.6) satisfy

$$
\mathcal{I}\left(q_{i}, p_{j}\right)=W_{i+1}^{T} V_{j+1}=\delta_{i j} I_{k}, \quad 0 \leq i, j \leq N
$$


Proof. We have for $0 \leq i, j \leq N$ that

$$
\begin{aligned}
\delta_{i j} I_{k}=W_{i+1}^{T} V_{j+1} & =\left(\sum_{s=0}^{i}\left(A^{T}\right)^{s} W_{1} D_{s}^{(i)}\right)^{T}\left(\sum_{t=0}^{j} A^{t} V_{1} C_{t}^{(j)}\right) \\
& =\sum_{s=0}^{i} \sum_{t=0}^{j}\left(D_{s}^{(i)}\right)^{T} W_{1}^{T} A^{s+t} V_{1} C_{t}^{(j)} \\
& =\sum_{s=0}^{i} \sum_{t=0}^{j}\left(D_{s}^{(i)}\right)^{T} \mathcal{I}\left(\lambda^{s+t}\right) C_{t}^{(j)} \\
& =\sum_{s=0}^{i} \sum_{t=0}^{j}\left(D_{s}^{(i)}\right)^{T} \mathcal{I}\left(\bar{\lambda}^{s}, \lambda^{t}\right) C_{t}^{(j)} \\
& =\sum_{s=0}^{i} \sum_{t=0}^{j} \mathcal{I}\left(\bar{\lambda}^{s} D_{s}^{(i)}, \lambda^{t} C_{t}^{(j)}\right) \\
& =\mathcal{I}\left(\sum_{s=0}^{i} \bar{\lambda}^{s} D_{s}^{(i)}, \sum_{t=0}^{j} \lambda^{t} C_{t}^{(j)}\right)=\mathcal{I}\left(q_{i}, p_{j}\right),
\end{aligned}
$$

where $\mathcal{I}$ in (4.7) is defined by (3.16), in (4.8) and below by (3.27).

One can show that, under the assumptions following (4.5), the matrix polynomials $p_{j}$ and $q_{i}$ satisfy the recursion relation (3.17) with the matrix recursion coefficients $\Delta_{j}, \Gamma_{j}$, and $\Omega_{j}$ defined by the nonsymmetric Lanczos recursions (4.4).

We are in a position to describe properties of the eigenvalues and eigenvectors of the block tridiagonal matrix $J_{N}$ in (4.5). This extends Theorem 2 to the nonsymmetric setting.

THEOREM 4. Let the matrix $J_{N}$ be defined by (4.5) and let $P_{N}$ and $Q_{N}$ be given by (3.18) with the polynomials $p_{i}$ and $q_{i}$ from (4.6). Then the following properties hold:

(1) The eigenvalues of $J_{N}$ are the zeros of both $\operatorname{det}\left[p_{N}(\lambda)\right]$ and $\operatorname{det}\left[q_{N}(\lambda)\right]$.

(2) The unit right eigenvector $\boldsymbol{y}_{r}^{(N)}$ of $J_{N}$ corresponding to the eigenvalue $\theta_{r}^{(N)}$ is given by $Q_{N}^{T}\left(\theta_{r}^{(N)}\right) \boldsymbol{u}_{r}^{(N)}$, where $\boldsymbol{u}_{r}^{(N)}$ consists of the first $k$ components of $\boldsymbol{y}_{r}^{(N)}$. Moreover, $q_{N}^{T}\left(\theta_{r}^{(N)}\right) \boldsymbol{u}_{r}^{(N)}=\mathbf{0}$.

(3) The unit right eigenvector $\boldsymbol{z}_{r}^{(N)}$ of $J_{N}^{T}$ corresponding to the eigenvalue $\theta_{r}^{(N)}$ is given by $P_{N}^{T}\left(\theta_{r}^{(N)}\right) \boldsymbol{v}_{r}^{(N)}$, where $\boldsymbol{v}_{r}^{(N)}$ consists of the first $k$ components of $\boldsymbol{z}_{r}^{(N)}$. Further, $p_{N}^{T}\left(\theta_{r}^{(N)}\right) \boldsymbol{v}_{r}^{(N)}=\mathbf{0 .}$.

Proof. Our proof is inspired by the proof of [18, Theorem 1.1]. We establish the relation between the zeros of $\operatorname{det}\left[q_{N}(\lambda)\right]$ and the eigenvalues of $J_{N}$ in (1), as well as (2). The remainder of the proof follows similarly and, therefore, is omitted. 
Suppose that $J_{N} \boldsymbol{y}=\theta \boldsymbol{y}$ for $\boldsymbol{y} \neq \mathbf{0}$, and write $\boldsymbol{y}^{T}=\left[\boldsymbol{y}_{1}^{T}, \ldots, \boldsymbol{y}_{N}^{T}\right]$, where $\boldsymbol{y}_{i} \in \mathbb{C}^{k}$ for $1 \leq i \leq N$. Notice that

$$
\begin{aligned}
\Omega_{1} \boldsymbol{y}_{1}+\Delta_{1}^{T} \boldsymbol{y}_{2} & =\theta \boldsymbol{y}_{1}, \\
& \vdots \\
\Gamma_{i-1} \boldsymbol{y}_{i-1}+\Omega_{i} \boldsymbol{y}_{i}+\Delta_{i}^{T} \boldsymbol{y}_{i+1} & =\theta \boldsymbol{y}_{i}, \\
& \vdots \\
\Gamma_{N-1} \boldsymbol{y}_{N-1}+\Omega_{N} \boldsymbol{y}_{N} & =\theta \boldsymbol{y}_{N} .
\end{aligned}
$$

Since $\Omega_{i}, \Gamma_{i}, \Delta_{i}$ are the matrix recurrence coefficients for the polynomials $q_{i}$, we obtain by induction that

$$
\begin{aligned}
\boldsymbol{y}_{i+1} & =q_{i}^{T}(\theta) \boldsymbol{y}_{1}, \quad 0 \leq i \leq N-1, \\
\mathbf{0} & =q_{N}^{T}(\theta) \boldsymbol{y}_{1},
\end{aligned}
$$

where we have used that the matrices $\Delta_{i}$ are invertible for $1 \leq i \leq N$. Since $\boldsymbol{y} \neq \mathbf{0}$, it follows that $\boldsymbol{y}_{1} \neq \mathbf{0}$ and, therefore, $\operatorname{det}\left[q_{N}(\theta)\right]=0$. This also establishes (2).

It remains to show that every zero of $\operatorname{det}\left[q_{N}(\theta)\right]$ is an eigenvalue of $J_{N}$. Suppose that $\operatorname{det}\left[q_{N}(\theta)\right]=0$. Then there is a vector $\boldsymbol{u} \in \mathbb{C}^{N k} \backslash\{\mathbf{0}\}$ such that $\boldsymbol{u}^{T} q_{N}(\theta)=\mathbf{0}$. By (3.19), this implies that

$$
J_{N} Q_{N}^{T}(\theta) \boldsymbol{u}=\theta Q_{N}^{T}(\theta) \boldsymbol{u} .
$$

Since $q_{0}(\theta) \equiv I_{k}$, the vector $Q_{N}^{T}(\theta) \boldsymbol{u}$ is nonzero and is, thus, a right eigenvector of $J_{N}$ associated with the eigenvalue $\theta$. This establishes part (1) regarding $\operatorname{det}\left[q_{N}(\theta)\right]$.

\section{Block Gauss quadrature rules.}

5.1. The symmetric problem $\boldsymbol{W}^{\boldsymbol{T}} \boldsymbol{f}(\boldsymbol{A}) \boldsymbol{W}$. We show that the quadrature rule $\mathcal{G}_{N}$ defined by (3.6) and used to approximate (1.1) when $A=A^{T}$ is exact for all polynomials in $\mathbb{P}^{2 N-1}$. Our proof is formulated entirely in terms of linear algebra and is shorter than existing proofs. The result was established in [28] and at about the same time extended in [45] to a more general (not necessarily discrete) class of matrix measures.

THEOREM 5. Let the function $\mathcal{I}$ be defined by (3.2) and the associated quadrature rule $\mathcal{G}_{N}$ by (3.6). Then $\mathcal{G}_{N} f=\mathcal{I} f$ for all $f \in \mathbb{P}^{2 N-1}$.

Proof. We first recall that the polynomials in the sets $\mathbb{P}^{j}$ have scalar coefficients. For fixed $N$, one can show by "induction" on the degree of $p$ that

$$
p(A) X_{1}=X^{(N)} p\left(J_{N}\right) E_{1}, \quad p \in \mathbb{P}^{N-1},
$$

where $X^{(N)}:=\left[X_{1}, \ldots, X_{N}\right]$. Moreover,

$$
\left(X^{(N)}\right)^{T} q(A) X_{1}=q\left(J_{N}\right) E_{1}, \quad q \in \mathbb{P}^{N} .
$$

Let $f \in \mathbb{P}^{2 N-1}$. We may factor $f=p q$, where $p \in \mathbb{P}^{N-1}$ and $q \in \mathbb{P}^{N}$. Recalling that $X_{1}=W$, we obtain

$$
\begin{aligned}
\mathcal{I} f & =W^{T} f(A) W=\left[X_{1}^{T} p(A)\right] q(A) X_{1} \\
& =\left[E_{1}^{T} p\left(J_{N}\right)\left(X^{(N)}\right)^{T}\right] q(A) X_{1}=E_{1}^{T} p\left(J_{N}\right)\left[\left(X^{(N)}\right)^{T} q(A) X_{1}\right] \\
& =E_{1}^{T} p\left(J_{N}\right)\left[q\left(J_{N}\right) E_{1}\right]=E_{1}^{T} f\left(J_{N}\right) E_{1}=\mathcal{G}_{N} f .
\end{aligned}
$$


We end this section with a generalization of Theorem 5 that will be needed in the following section. This result is also shown in [45]. We include the proof because it will be referred to below.

Corollary 6. Let $p(\lambda)$ and $q(\lambda)$ be polynomials with $k \times k$ matrix coefficients, and let $\mathcal{I}$ and $\mathcal{G}_{N}$ be defined by (3.14) and (3.15), respectively. Then $\mathcal{G}_{N}(p, q)=\mathcal{I}(p, q)$ when $\operatorname{deg} p+\operatorname{deg} q \leq 2 N-1$.

Proof. The proof is related to the proof of Theorem 3. Let

$$
p(\lambda)=\sum_{s=0}^{i} \lambda^{s} C_{s}, \quad q(\lambda)=\sum_{t=0}^{j} \lambda^{t} D_{t},
$$

with $i+j \leq 2 N-1$. By Theorem 5 , we have

$$
\begin{aligned}
\mathcal{G}_{N}(p, q) & =\mathcal{G}_{N}\left(\sum_{s=0}^{i} \lambda^{s} C_{s}, \sum_{t=0}^{j} \lambda^{t} D_{t}\right)=\sum_{s=0}^{i} \sum_{t=0}^{j} C_{s}^{T} \mathcal{G}_{N}\left(\lambda^{s}, \lambda^{t}\right) D_{m} \\
& =\sum_{s=0}^{i} \sum_{t=0}^{j} C_{s}^{T} \mathcal{G}_{N}\left(\lambda^{s+t}\right) D_{t}=\sum_{s=0}^{i} \sum_{t=0}^{j} C_{s}^{T} \mathcal{I}\left(\lambda^{s+t}\right) D_{t} \\
& =\sum_{s=0}^{i} \sum_{t=0}^{j} C_{s}^{T} \mathcal{I}\left(\lambda^{s}, \lambda^{t}\right) D_{t}=\sum_{s=0}^{i} \sum_{t=0}^{j} \mathcal{I}\left(\lambda^{s} C_{s}, \lambda^{t} D_{t}\right) \\
& =\mathcal{I}\left(\sum_{s=0}^{i} \lambda^{s} C_{s}, \sum_{t=0}^{j} \lambda^{t} D_{t}\right)=\mathcal{I}(p, q) .
\end{aligned}
$$

5.2. The nonsymmetric problem $\boldsymbol{W}^{\boldsymbol{T}} \boldsymbol{f}(\boldsymbol{A}) \boldsymbol{V}$. We establish results related to functions (1.2) that are analogous to those of the previous subsection.

TheOREM 7. Let the function $\mathcal{I}$ be defined by (3.16) and the associated quadrature rule $\mathcal{G}_{N}$ by (3.21). Then $\mathcal{G}_{N} f=\mathcal{I} f$ for all $f \in \mathbb{P}^{2 N-1}$.

Proof. The proof is similar to that of Theorem 5. Thus, we first show by "induction" on the degree of $p$ that for fixed $N$,

$$
\begin{aligned}
p\left(A^{T}\right) W_{1} & =W^{(N)} p\left(J_{N}^{T}\right) E_{1}, & & p \in \mathbb{P}^{N-1}, \\
\left(W^{(N)}\right)^{T} q(A) V_{1} & =q\left(J_{N}\right) E_{1}, & & q \in \mathbb{P}^{N},
\end{aligned}
$$

where $W^{(N)}:=\left[W_{1}, \ldots, W_{N}\right]$.

Let $f \in \mathbb{P}^{2 N-1}$. Factoring $f=p q$, where $p \in \mathbb{P}^{N-1}$ and $q \in \mathbb{P}^{N}$, yields similarly as in the proof of Theorem 5 that

$$
\mathcal{I} f=W_{1}^{T} p(A) q(A) V_{1}=E_{1}^{T} p\left(J_{N}\right) q\left(J_{N}\right) E_{1}=E_{1}^{T} f\left(J_{N}\right) E_{1}=\mathcal{G}_{N} f .
$$

We end this section with a generalization of Theorem 7 which we will apply below.

COROLlary 8. Let $p(\lambda)$ and $q(\lambda)$ be polynomials with $k \times k$ matrix coefficients, and let $\mathcal{I}(p, q)$ and $\mathcal{G}_{N}(p, q)$ be defined by (3.27) and (3.28), respectively. Then $\mathcal{G}_{N}(p, q)=\mathcal{I}(p, q)$ holds when $\operatorname{deg} p+\operatorname{deg} q \leq 2 N-1$.

Proof. The proof is similar to that of Corollary 6. 


\section{Anti-Gauss quadrature rules.}

6.1. The symmetric problem $\boldsymbol{W}^{\boldsymbol{T}} \boldsymbol{f}(\boldsymbol{A}) \boldsymbol{W}$. In this section, we show how the matrix $\tilde{J}_{N+1}$, defined by (3.12) and associated with the $(N+1)$-block anti-Gauss quadrature rule (3.9), can be obtained, with almost no work, from the matrix $J_{N+1}$ defined by (3.5), with $N$ replaced by $N+1$, associated with the $(N+1)$-block Gauss quadrature rule determined by (3.6) with $N$ replaced by $N+1$.

It follows from (3.3) that the coefficient matrices $\Omega_{i}$ and $\Gamma_{i}$ associated with the block Gauss rule (3.6), with $N$ replaced by $N+1$, are given by

$$
\Omega_{i}=\mathcal{I}\left(p_{i-1}, \lambda p_{i-1}\right), \quad \Gamma_{i}=\mathcal{I}\left(p_{i}, \lambda p_{i-1}\right) .
$$

Similarly, we obtain from (3.11) that the coefficients $\tilde{\Omega}_{i}$ and $\tilde{\Gamma}_{i}$ associated with the block anti-Gauss rule (3.9) satisfy

$$
\tilde{\Omega}_{i}=\left(2 \mathcal{I}-\mathcal{G}_{N}\right)\left(\tilde{p}_{i-1}, \lambda \tilde{p}_{i-1}\right), \quad \tilde{\Gamma}_{i}=\left(2 \mathcal{I}-\mathcal{G}_{N}\right)\left(\tilde{p}_{i}, \lambda \tilde{p}_{i-1}\right) .
$$

Therefore, the recursions (3.3) and (3.11), together with (3.9) and Corollary 6, imply that

$$
\begin{aligned}
\tilde{\Omega}_{i}=\Omega_{i}, & 1 \leq i \leq N, \\
\tilde{\Gamma}_{i}=\Gamma_{i}, & 1 \leq i \leq N-1, \\
\tilde{p}_{i}=p_{i}, & 0 \leq i \leq N-1 .
\end{aligned}
$$

It follows that

$$
\begin{aligned}
\tilde{p}_{N} \tilde{\Gamma}_{N} & =\lambda I_{k} \tilde{p}_{N-1}-\tilde{p}_{N-1} \tilde{\Omega}_{N}-\tilde{p}_{N-2} \tilde{\Gamma}_{N-1}^{T} \\
& =\lambda I_{k} p_{N-1}-p_{N-1} \Omega_{N}-p_{N-2} \Gamma_{N-1}^{T}=p_{N} \Gamma_{N} .
\end{aligned}
$$

Hence,

$$
\begin{aligned}
\tilde{\Gamma}_{N} & =\left(2 \mathcal{I}-\mathcal{G}_{N}\right)\left(\tilde{p}_{N}, \lambda \tilde{p}_{N-1}\right)=2 \mathcal{I}\left(\tilde{p}_{N}, \lambda \tilde{p}_{N-1}\right)-\mathcal{G}_{N}\left(\tilde{p}_{N}, \lambda \tilde{p}_{N-1}\right) \\
& =2\left(\Gamma_{N} \tilde{\Gamma}_{N}^{-1}\right)^{T} \mathcal{I}\left(p_{N}, \lambda p_{N-1}\right)=2\left(\Gamma_{N} \tilde{\Gamma}_{N}^{-1}\right)^{T} \Gamma_{N},
\end{aligned}
$$

where $\mathcal{G}_{N}\left(\tilde{p}_{N}, \lambda \tilde{p}_{N-1}\right)=\mathbf{0}$, because in view of Theorem 2 , we have

$$
\tilde{p}_{N}^{T}\left(\theta_{r}^{(N)}\right) \boldsymbol{u}_{r}^{(N)}=\left(\Gamma_{N} \tilde{\Gamma}_{N}^{-1}\right)^{T} p_{N}^{T}\left(\theta_{r}^{(N)}\right) \boldsymbol{u}_{r}^{(N)}=\mathbf{0}, \quad 1 \leq r \leq k N .
$$

We conclude that $\tilde{\Gamma}_{N}^{T} \tilde{\Gamma}_{N}=2 \Gamma_{N}^{T} \Gamma_{N}$. Recall that the matrices $\Gamma_{N}$ and $\tilde{\Gamma}_{N}$ are assumed to be invertible, and are chosen to have positive diagonal entries. Therefore,

$$
\tilde{\Gamma}_{N}=\sqrt{2} \Gamma_{N}
$$

because the symmetric positive definite matrix $2 \Gamma_{N}^{T} \Gamma_{N}$ has a unique Cholesky factorization $C^{T} C$ with an upper-triangular factor $C$, whose diagonal is strictly positive.

We turn to the entry $\tilde{\Omega}_{N+1}$. It follows from (6.2) that $\Gamma_{N} \tilde{\Gamma}_{N}^{-1}=(1 / \sqrt{2}) I_{k}$, which, in view of $(6.1)$, implies that $\tilde{p}_{N}=(1 / \sqrt{2}) p_{N}$. Therefore,

$$
\tilde{\Omega}_{N+1}=\left(2 \mathcal{I}-\mathcal{G}_{N}\right)\left(\tilde{p}_{N}, \lambda \tilde{p}_{N}\right)=\mathcal{I}\left(p_{N}, \lambda p_{N}\right)=\Omega_{N+1} .
$$

In conclusion, the matrix $\tilde{J}_{N+1}$ associated with the $(N+1)$-block anti-Gauss rule can be obtained from the matrix $J_{N+1}$ associated with the $(N+1)$-block Gauss rule by multiplying $\Gamma_{N}$ by $\sqrt{2}$. 
6.2. The nonsymmetric problem $\boldsymbol{W}^{\boldsymbol{T}} \boldsymbol{f}(\boldsymbol{A}) \boldsymbol{V}$. We show how the matrix $\tilde{J}_{N+1}$ given by (3.25) and associated with the nonsymmetric $(N+1)$-block anti-Gauss rule defined by (3.23) can be determined, with almost no work, from the matrix $J_{N+1}$, given by (3.20) with $N$ replaced by $N+1$, associated with the $(N+1)$-block nonsymmetric Gauss rule (3.21) with $N$ replaced by $N+1$. The following discussion is analogous to that of the previous subsection.

We obtain from (3.17) and (3.24) that the coefficients $\Omega_{i}, \Gamma_{i}$, and $\Delta_{i}$ associated with nonsymmetric block Gauss rules and the coefficients $\tilde{\Omega}_{i}, \tilde{\Gamma}_{i}$, and $\tilde{\Delta}_{i}$ of nonsymmetric block anti-Gauss rules are given by

$$
\begin{aligned}
\Omega_{i} & =\mathcal{I}\left(q_{i-1}, \lambda p_{i-1}\right), & \tilde{\Omega}_{i} & =\left(2 \mathcal{I}-\mathcal{G}_{N}\right)\left(\tilde{q}_{i-1}, \lambda \tilde{p}_{i-1}\right), \\
\Gamma_{i} & =\mathcal{I}\left(q_{i}, \lambda p_{i-1}\right), & \tilde{\Gamma}_{i} & =\left(2 \mathcal{I}-\mathcal{G}_{N}\right)\left(\tilde{q}_{i}, \lambda \tilde{p}_{i-1}\right), \\
\Delta_{i}^{T} & =\mathcal{I}\left(q_{i-1}, \lambda p_{i}\right), & \tilde{\Delta}_{i}^{T} & =\left(2 \mathcal{I}-\mathcal{G}_{N}\right)\left(\tilde{q}_{i-1}, \lambda \tilde{p}_{i}\right),
\end{aligned}
$$

where $\mathcal{I}$ and $\mathcal{G}_{N}$ are defined by (3.27) and (3.28), respectively. Hence, the recursions (3.17) and (3.24), together with (3.23) and Corollary 8, yield

$$
\begin{aligned}
& \tilde{\Omega}_{i}=\Omega_{i}, \quad 1 \leq i \leq N, \\
& \tilde{\Gamma}_{i}=\Gamma_{i}, \quad \tilde{\Delta}_{i}=\Delta_{i}, \quad 1 \leq i \leq N-1, \\
& \tilde{p}_{i}=p_{i}, \quad \tilde{q}_{i}=q_{i}, \quad 0 \leq i \leq N-1,
\end{aligned}
$$

from which we conclude that

$$
\begin{aligned}
\tilde{p}_{N} \tilde{\Gamma}_{N} & =\lambda \tilde{p}_{N-1}-\tilde{p}_{N-1} \tilde{\Omega}_{N}-\tilde{p}_{N-2} \tilde{\Delta}_{N-1}^{T} \\
& =\lambda p_{N-1}-p_{N-1} \Omega_{N}-p_{N-2} \Delta_{N-1}^{T}=p_{N} \Gamma_{N}, \\
\tilde{q}_{N} \tilde{\Delta}_{N} & =\lambda \tilde{q}_{N-1}-\tilde{q}_{N-1} \tilde{\Omega}_{N}^{T}-\tilde{q}_{N-2} \tilde{\Gamma}_{N-1}^{T} \\
& =\lambda q_{N-1}-q_{N-1} \Omega_{N}^{T}-q_{N-2} \Gamma_{N-1}^{T}=q_{N} \Delta_{N} .
\end{aligned}
$$

Thus,

$$
\begin{aligned}
\tilde{\Gamma}_{N} & =\left(2 \mathcal{I}-\mathcal{G}_{N}\right)\left(\tilde{q}_{N}, \lambda \tilde{p}_{N-1}\right)=2 \mathcal{I}\left(\tilde{q}_{N}, \lambda \tilde{p}_{N-1}\right)-\mathcal{G}_{N}\left(\tilde{q}_{N}, \lambda \tilde{p}_{N-1}\right) \\
& =2\left(\Delta_{N} \tilde{\Delta}_{N}^{-1}\right)^{T} \mathcal{I}\left(q_{N}, \lambda p_{N-1}\right)=2\left(\Delta_{N} \tilde{\Delta}_{N}^{-1}\right)^{T} \Gamma_{N},
\end{aligned}
$$

where we have used that $\mathcal{G}_{N}\left(\tilde{q}_{N}, \lambda \tilde{p}_{N-1}\right)=\mathbf{0}$. This follows from the fact that

$$
\tilde{q}_{N}^{T}\left(\theta_{r}^{(N)}\right) \boldsymbol{u}_{r}^{(N)}=\left(\Delta_{N} \tilde{\Delta}_{N}^{-1}\right)^{T} q_{N}^{T}\left(\theta_{r}^{(N)}\right) \boldsymbol{u}_{r}^{(N)}=\mathbf{0}, \quad 1 \leq r \leq k N,
$$

which is a consequence of Theorem 4. Therefore, $\tilde{\Delta}_{N}^{T} \tilde{\Gamma}_{N}=2 \Delta_{N}^{T} \Gamma_{N}$. There is some freedom in choosing the blocks $\tilde{\Gamma}_{N}$ and $\tilde{\Delta}_{N}$. We will choose

$$
\tilde{\Gamma}_{N}=\sqrt{2} \Gamma_{N}, \quad \tilde{\Delta}_{N}=\sqrt{2} \Delta_{N}
$$

To show that $\tilde{\Omega}_{N+1}=\Omega_{N+1}$, we first observe that in view of (6.4) we have

$$
\Gamma_{N} \tilde{\Gamma}_{N}^{-1}=\Delta_{N} \tilde{\Delta}_{N}^{-1}=(1 / \sqrt{2}) I_{k},
$$

which by $(6.3)$ implies that $\tilde{p}_{N}=(1 / \sqrt{2}) p_{N}$ and $\tilde{q}_{N}=(1 / \sqrt{2}) q_{N}$. Therefore,

$$
\tilde{\Omega}_{N+1}=\left(2 \mathcal{I}-\mathcal{G}_{N}\right)\left(\tilde{q}_{N}, \lambda \tilde{p}_{N}\right)=\mathcal{I}\left(q_{N}, \lambda p_{N}\right)=\Omega_{N+1} .
$$

Thus, similarly as in subsection 6.1 , the matrix $\tilde{J}_{N+1}$ given by $(3.25)$ can be obtained from the matrix $J_{N+1}$ associated with the nonsymmetric $(N+1)$-block Gauss rule by multiplying the last off-diagonal blocks $\Gamma_{N}$ and $\Delta_{N}$ by $\sqrt{2}$. 
7. Bounds by standard Gauss-type rules. This section provides a few more details about the technique advocated by Golub and Meurant [28, 29] for computing bounds for expressions of the form

$$
\mathcal{I} f=\boldsymbol{w}^{T} f(A) \boldsymbol{v}
$$

when the matrix $A \in \mathbb{R}^{m \times m}$ is symmetric and $\boldsymbol{w}, \boldsymbol{v} \in \mathbb{R}^{m}$. We pay particular attention to the function $f(A)=\exp (A)$, which we will use in computed examples of the following section.

Let the function $f$ be analytic in the convex hull $\left[\lambda_{\min }, \lambda_{\max }\right]$ of the spectrum of $A$ and have derivatives $f^{(j)}, j=1,2, \ldots$, of constant sign there. Let $\boldsymbol{v}=\boldsymbol{w}$. If $f^{(2 n)} \geq 0$ on $\left[\lambda_{\min }, \lambda_{\max }\right]$, then the remainder formula for the $n$-point (standard) Gauss quadrature rule $\mathcal{G}_{n}$ shows that $\mathcal{G}_{n} f \leq \mathcal{I} f$; see, e.g., [5, 14, 28, 29] for details. If, in addition, $f^{(2 n+2)} \geq 0$ on $\left[\lambda_{\min }, \lambda_{\max }\right]$, then one can show that, generically, $\mathcal{G}_{n} f<$ $\mathcal{G}_{n+1} f$; see, e.g., [38] for this result and related ones for Gauss-Radau quadrature rules. Turning to the latter type of rules, let $a \leq \lambda_{\min }$ and assume that $f$ is analytic in the interval $\left[a, \lambda_{\max }\right]$. Moreover, let $f^{(2 n+1)} \leq 0$ on this interval. Then the remainder formula for the $(n+1)$-point Gauss-Radau rule $\mathcal{R}_{n+1, a}$ with a fixed node at $a$ shows that $\mathcal{R}_{n+1, a} f \geq \mathcal{I} f$. Thus, the Gauss and Gauss-Radau approximations bracket $\mathcal{I} f$. Similarly, let $b \geq \lambda_{\max }$ and assume that $f$ is analytic in the interval $\left[\lambda_{\min }, b\right]$. If $f^{(2 n+1)} \geq 0$ on $\left[\lambda_{\min }, b\right]$, then the $(n+1)$-point Gauss-Radau rule $\mathcal{R}_{n+1, b}$ with a fixed node at $b$ satisfies $\mathcal{R}_{n+1, b} f \geq \mathcal{I} f$. Hence, when the derivatives of $f$ are of constant sign on the interval $[a, b]$, suitable combinations of Gauss and Gauss-Radau rules bound the value of the desired integral. Similar results hold for Gauss-Lobatto quadrature rules; we refer to $[28,29,38]$ for proofs.

We turn to the case when $\boldsymbol{w} \neq \boldsymbol{v}$. Bounds for the quantity (7.1) can be derived, for example, by first computing bounds for $(\boldsymbol{w}+\boldsymbol{v})^{T} f(A)(\boldsymbol{w}+\boldsymbol{v})$ and $(\boldsymbol{w}-\boldsymbol{v})^{T} f(A)(\boldsymbol{w}-\boldsymbol{v})$, and then using the relation

$$
\boldsymbol{w}^{T} f(A) \boldsymbol{v}=\frac{1}{4}\left((\boldsymbol{w}+\boldsymbol{v})^{T} f(A)(\boldsymbol{w}+\boldsymbol{v})-(\boldsymbol{w}-\boldsymbol{v})^{T} f(A)(\boldsymbol{w}-\boldsymbol{v})\right) .
$$

This approach is suggested in $[28,29]$. In the context of network analysis, one is often interested in computing the $f$-subgraph centrality (2.3) for important nodes, i.e., for nodes for which this quantity is large, and in determining the $f$-communicability for these nodes; see section 2 . If bounds for the $f$-subgraph centralities for the nodes $i$ and $j$ already are available, then it is convenient to use these bounds to determine bounds for the $f$-communicability between these nodes. For example, if

$$
\begin{aligned}
& L_{1} \leq \frac{1}{\sqrt{2}}\left(\boldsymbol{e}_{i}+\boldsymbol{e}_{j}\right)^{T} f(A) \frac{1}{\sqrt{2}}\left(\boldsymbol{e}_{i}+\boldsymbol{e}_{j}\right) \leq U_{1}, \\
& L_{2} \leq \boldsymbol{e}_{i}^{T} f(A) \boldsymbol{e}_{i} \leq U_{2}, \\
& L_{3} \leq \boldsymbol{e}_{j}^{T} f(A) \boldsymbol{e}_{j} \leq U_{3},
\end{aligned}
$$

then upper and lower bounds for $\boldsymbol{e}_{i}^{T} f(A) \boldsymbol{e}_{j}$ are given by $U_{1}-L_{2} / 2-L_{3} / 2$ and $L_{1}-U_{2} / 2-U_{3} / 2$, respectively. Since the bounds $L_{i}$ and $U_{i}$ for $i=2,3$ are assumed to already be available, this approach only requires the additional computation of the bounds $L_{1}$ and $U_{1}$.

The main advantage of the approach described above is that it yields bounds for (7.1). However, the computational effort required may be much larger than when block methods are used. Suppose that bounds are desired for each element of the 
leading $k \times k$ submatrix of $f(A)$, i.e., of $\mathcal{I} f=W^{T} f(A) W$ with $W=\left[\boldsymbol{e}_{1}, \ldots, \boldsymbol{e}_{k}\right]$. Using the approach of this section requires $k(k+1) / 2$ partial Lanczos decompositions with $\boldsymbol{w}=\boldsymbol{e}_{i}$ for $1 \leq i \leq k$ and $\boldsymbol{w}=\left(\boldsymbol{e}_{i}+\boldsymbol{e}_{j}\right) / \sqrt{2}$ for $1 \leq i<j \leq k$. Approximations of these bounds can be computed by the block methods described in section 3.1. They require the evaluation of a single partial block Lanczos decomposition with block-size $k$. If the Lanczos method with block-size one requires about the same number of steps as the block Lanczos method with block-size $k$, then the count of matrix-vector products scales with $k$ for the block method, while it scales with $k^{2}$ when the blocksize is one. Here we count the product of the matrix $A$ with a block-vector with $k$ columns as $k$ matrix-vector products.

As pointed out in subsection 4.2, when we apply the nonsymmetric Lanczos method with a sparse initial vector, such as the axis vector $\boldsymbol{e}_{i}$, to a nonsymmetric adjacency matrix associated with a directed graph, breakdown commonly takes place. Let $\boldsymbol{c}=[1, \ldots, 1]^{T}$ and let $\boldsymbol{c}_{i}=\boldsymbol{c}-\boldsymbol{e}_{i}$. The nonsymmetric block Lanczos method with initial orthonormal block $\left[\boldsymbol{e}_{i}, \boldsymbol{c}_{i} /\left\|\boldsymbol{c}_{i}\right\|\right]$ applied to a nonsymmetric adjacency matrix rarely breaks down. Therefore, block Lanczos methods are essential for the investigation of directed graphs.

8. Numerical examples. We illustrate in this section the application of block Gauss and anti-Gauss quadrature rules to the estimation of certain functions of symmetric or nonsymmetric adjacency matrices. All computations were carried out with MATLAB version 8.1 (R2013a) 64-bit for Linux, in double precision arithmetic, on an Intel Core i7-860 computer with 8 GB RAM. The following numerical examples illustrate the performance of block Gauss and block anti-Gauss quadrature rules when applied to integrate the exponential function. The matrices for most examples are adjacency matrices for undirected or directed networks that arise in real-world applications and are publicly available.

We will approximate $\mathcal{I} f$ by

$$
F_{N}:=\mathcal{A}_{N+1} f=\frac{1}{2}\left(\mathcal{G}_{N} f+\mathcal{H}_{N+1} f\right) ;
$$

cf. (3.10). Seeking to determine each entry of $\mathcal{I} f$ with an approximate relative tolerance $\tau$, we terminate the symmetric and nonsymmetric block Lanczos methods at iteration $N$, where $N$ is the smallest integer such that

$$
T_{N}:=\frac{1}{2} \frac{\left\|\mathcal{G}_{N} f-\mathcal{H}_{N+1} f\right\|_{\max }}{\left\|F_{N}\right\|_{\max }}<\tau,
$$

with $\|B\|_{\max }:=\max _{1 \leq i, j \leq k}\left|B_{i j}\right|$, and then accept (8.1) as our approximation of $\mathcal{I} f$. In all experiments of this section, we let $\tau=10^{-3}$.

Assume that

$$
\min \left\{\left[\mathcal{G}_{N} f\right]_{i j},\left[\mathcal{H}_{N+1} f\right]_{i j}\right\} \leq[\mathcal{I} f]_{i j} \leq \max \left\{\left[\mathcal{G}_{N} f\right]_{i j},\left[\mathcal{H}_{N+1} f\right]_{i j}\right\}
$$

Then

$$
\begin{aligned}
\left|\left[F_{N}-\mathcal{I} f\right]_{i j}\right| & =\left|\left[\frac{1}{2} \mathcal{G}_{N} f-\frac{1}{2} \mathcal{I} f\right]_{i j}+\left[\frac{1}{2} \mathcal{H}_{N+1} f-\frac{1}{2} \mathcal{I} f\right]_{i j}\right| \\
& \leq \frac{1}{2}\left|\left[\mathcal{G}_{N} f-\mathcal{I} f\right]_{i j}\right|+\frac{1}{2}\left|\left[\mathcal{H}_{N+1} f-\mathcal{I} f\right]_{i j}\right| \\
& =\frac{1}{2}\left|\left[\mathcal{G}_{N} f-\mathcal{H}_{N+1} f\right]_{i j}\right| .
\end{aligned}
$$


Therefore, if $\left[\mathcal{G}_{N} f\right]_{i j}$ and $\left[\mathcal{H}_{N+1} f\right]_{i j}$ bracket $[\mathcal{I} f]_{i j}$ for all $1 \leq i, j \leq k$, then $(8.2)$ implies that

$$
G_{N}:=\frac{\left\|F_{N}-\mathcal{I} f\right\|_{\max }}{\left\|F_{N}\right\|_{\max }}<\tau
$$

i.e., $F_{N}$ approximates $\mathcal{I} f$ elementwise with a relative error bounded by about $\tau$.

Computations with the function $f(A)=\exp (A)$ may give rise to overflow when the adjacency matrix $A$ is large. This can be avoided by spectrum shift in the following way. First, consider the case of a symmetric block tridiagonal matrix $J_{N}$. When evaluating (3.7), the shift $\mu$ is chosen to be the largest eigenvalue of $J_{N}$, and we evaluate the exponential of the shifted matrix $J_{N}-\mu I_{k N}$ using the spectral factorization of $J_{N}$. The factor $\exp (\mu)$ does not have to be computed. The computation of (3.13) is carried out similarly. For the nonsymmetric matrix $J_{N}$ defined by (3.20), we choose the maximal real part of the eigenvalues as shift $\mu$ and use the MATLAB function expm to evaluate the exponential of the shifted matrix $J_{N}-\mu I_{k N}$ in (3.22). We proceed similarly when evaluating (3.26). Spectrum shift is also applied in the evaluation of standard Gauss-type rules described in section 7 .

We have not experienced breakdown in any of the reported computations. However, breakdowns have been observed in some examples when the block size is larger than 5 .

8.1. Applications to undirected graphs. We present some examples that show the performance of block Gauss and anti-Gauss quadrature rules associated with the symmetric block Lanczos method. This method is applied to seven real-world undirected unweighted networks, some of which have been investigated numerically in [25]. The networks have the following properties:

Email (1133 nodes, 10902 edges) is a representation of e-mail interchanges between members of the University Rovira i Virgili (Tarragona), described in [32]. The data set is available at Alex Arena's web page [1].

Autobahn (1168 nodes, 2486 edges) describes the German highway system network. The nodes are German locations and the edges are highways connecting them. It is available at $[9]$.

Yeast (2114 nodes, 4480 edges) describes the protein interaction network for yeast. Each edge represents an interaction between two proteins [35, 46]. The data set was originally included in the Notre Dame Networks Database, and it is now available at [4].

Power (4941 nodes, 13188 edges) is an undirected unweighted representation of the topology of the western states power grid of the United States, compiled by Watts and Strogatz [49]. The original data set was made available at the web site of Watts at Columbia University, and now can be found at [43].

Internet (22963 nodes, 96872 edges) is a symmetrized snapshot of the structure of the Internet at the level of autonomous systems, reconstructed from BGP (Border Gateway Protocol) tables posted by the University of Oregon Route Views Project. This snapshot was created by Mark Newman from data for July 22, 2006 [43].

Collaboration (40421 nodes, 351304 edges) is the collaboration network of scientists who submitted preprints to the condensed matter archive at www.arxiv.org [42] between January 1, 1995, and March 31, 2005. The original network is weighted, here we consider an unweighted version [43]. 
Facebook (63731 nodes, 1545686 edges) is the largest example we consider. It describes all the user-to-user links (friendships) of the Facebook New Orleans network. It was studied in [48], and the data set is available at [40].

We are interested in computing the $f$-subgraph centrality of $k$ specified nodes, as well as the $f$-communicability between each pair of nodes, for a total of $k(k+1) / 2 \mathrm{nu}$ merical quantities. We may assume that the nodes of interest are the nodes 1 through $k$. We, therefore, seek to approximate $\mathcal{I} f=W^{T} f(A) W$ with $W:=\left[\boldsymbol{e}_{1}, \ldots, \boldsymbol{e}_{k}\right]$.

To investigate whether for each $N$ the entries $\left[\mathcal{G}_{N} f\right]_{i j}$ and $\left[\mathcal{H}_{N+1} f\right]_{i j}$ bracket the quantity $[\mathcal{I} f]_{i j}$, we applied block Gauss and anti-Gauss rules to compute the centrality and communicability for five nodes of the first four networks. These networks are small enough to allow the evaluation of the matrix exponential by the MATLAB function expm. Since we cannot assume that the values returned by expm are exact, we checked that they are almost bounded by the computed quantities, i.e., whether

$$
\mathcal{L}-\sqrt{\epsilon_{M}}<[\operatorname{expm}(\mathrm{A})]_{i j}<\mathcal{U}+\sqrt{\epsilon_{M}}, \quad i, j=1, \ldots, 5,
$$

where $\mathcal{L}=\min \left\{\left[\mathcal{G}_{N} f\right]_{i j},\left[\mathcal{H}_{N+1} f\right]_{i j}\right\}, \mathcal{U}=\max \left\{\left[\mathcal{G}_{N} f\right]_{i j},\left[\mathcal{H}_{N+1} f\right]_{i j}\right\}$, and $\epsilon_{M} \simeq 2.2$. $10^{-16}$. These inequalities were satisfied for the first four networks, i.e., for all networks for which we could verify them.

Table 8.1 shows the execution time for the block Gauss and anti-Gauss quadrature rules, the scalar Gauss/Gauss-Radau quadrature rules described in section 7, and the expm function. We apply the quadrature rules to compute approximations of If $=W^{T} \exp (A) W$ with $W=\left[\boldsymbol{e}_{1}, \boldsymbol{e}_{2}, \boldsymbol{e}_{3}, \boldsymbol{e}_{4}, \boldsymbol{e}_{5}\right]$ and terminate the Lanczos and block Lanczos methods as soon as $T_{N}<10^{-3}$. The last networks of Table 8.1 are too large to allow the evaluation of the function expm. It can be seen that Lanczos methods can be applied to rather large networks, and that the block Lanczos method with block-size 5 is faster than the standard Lanczos method with block-size 1 .

Table 8.2 displays the number of matrix-vector product (MVP) evaluations and the error $G_{N}$ defined by (8.3) for both the scalar and the block case. The number of MVPs equals the number of steps of the Lanczos method when the block-size is one, and equals the product of the number of Lanczos steps and the block-size when the latter is larger than one. When evaluating $G_{N}$, we consider the value returned by expm to be exact. The error is only reported for the smallest networks and shows the termination criterion always to give approximations with the desired accuracy. Since the last three networks are too large to allow the evaluation of expm, we cannot report the errors for them.

To better illustrate the effect of the block-size on the execution time, we let the number of columns $k$ of $W$ increase. For each $k$ we approximate the entries of $W^{T} f(A) W$ both by the symmetric block Lanczos method with block-size $k$ and by

TABLE 8.1

Execution time, in seconds, for computing centralities of and communicabilities between five nodes of undirected networks.

\begin{tabular}{c|c|c|c|c|c}
\hline Matrix & Nodes & Edges & expm & Block-size 1 & Block-size 5 \\
\hline Email & 1133 & 10902 & $1.2 \mathrm{e}+01$ & $1.3 \mathrm{e}-01$ & $3.4 \mathrm{e}-02$ \\
Autobahn & 1168 & 2486 & $8.2 \mathrm{e}-01$ & $3.1 \mathrm{e}-01$ & $2.9 \mathrm{e}-02$ \\
Yeast & 2114 & 4480 & $1.0 \mathrm{e}+01$ & $9.6 \mathrm{e}-02$ & $3.3 \mathrm{e}-02$ \\
Power & 4941 & 13188 & $2.1 \mathrm{e}+01$ & $1.3 \mathrm{e}-01$ & $2.7 \mathrm{e}-02$ \\
Internet & 22963 & 96872 & - & $6.9 \mathrm{e}-01$ & $1.2 \mathrm{e}-01$ \\
Collab. & 40421 & 351384 & - & $1.6 \mathrm{e}+00$ & $3.5 \mathrm{e}-01$ \\
Facebook & 63731 & 1634180 & - & $4.5 \mathrm{e}+00$ & $6.0 \mathrm{e}-01$ \\
\hline
\end{tabular}


TABLE 8.2

Number of MVP evaluations and size of the error $G_{N}$ (8.3) for both block-sizes 1 and 5.

\begin{tabular}{c|c|c|cc|ccc}
\hline & & & \multicolumn{3}{|c|}{ Block-size 1 } & \multicolumn{3}{c}{ Block-size 5 } \\
Matrix & Nodes & Edges & MVP & $G_{N}$ & MVP & Steps & $G_{N}$ \\
\hline Email & 1133 & 10902 & 75 & $2.2 \mathrm{e}-05$ & 40 & 8 & $2.8 \mathrm{e}-06$ \\
Autobahn & 1168 & 2486 & 36 & $1.8 \mathrm{e}-05$ & 25 & 5 & $6.3 \mathrm{e}-07$ \\
Yeast & 2114 & 4480 & 35 & $8.1 \mathrm{e}-05$ & 35 & 7 & $2.6 \mathrm{e}-06$ \\
Power & 4941 & 13188 & 45 & $5.9 \mathrm{e}-06$ & 30 & 6 & $4.7 \mathrm{e}-07$ \\
Internet & 22963 & 96872 & 95 & - & 35 & 7 & - \\
Collab. & 40421 & 351384 & 100 & - & 50 & 10 & - \\
Facebook & 63731 & 1634180 & 102 & - & 50 & 10 & - \\
\hline
\end{tabular}
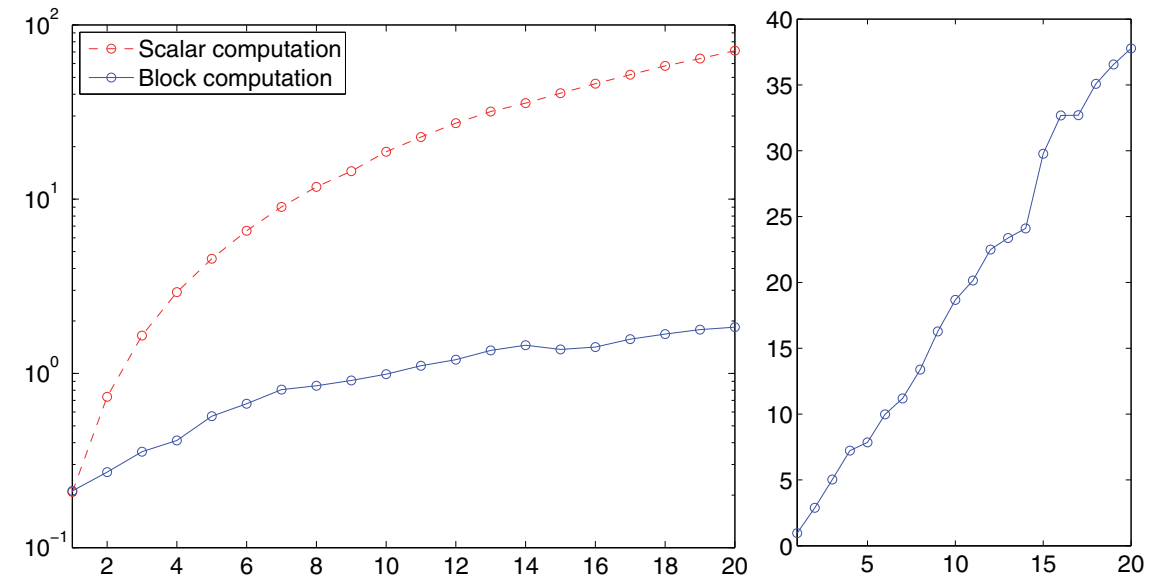

FIG. 8.1. Execution times for symmetric Lanczos methods with block-size 1 (top graph) and for block-size $k$ (bottom graph) as a function of $k$ when determining approximations of the entries of $W^{T} f(A) W$. The right panel shows the ratio between the timings.

$k(k+1) / 2$ applications of the standard Lanczos method with block-size one. The left panel of Figure 8.1 shows execution times, and the right panel the ratio between the execution times for block-sizes 1 and $k$. The speed-up of the block method is roughly linear as a function of the block-size.

A reason for the speed-up shown in Figure 8.1 is that both centralities and communicabilities were requested. We next investigate whether block methods are competitive when only centralities are desired. Figure 8.2 depicts execution times for this situation. The figure is analogous to Figure 8.1 and shows that for the computer used in our experiments, block-size 16 yields a speed-up of a little less than a factor of 2. Thus, also in the situation when only subgraph centralities are needed, the block method is competitive. In particular, it may be attractive to apply block Lanczos methods in the hybrid scheme for identifying the $k$ nodes of a network with the largest subgraph centrality proposed in [25]. This scheme first computes a low-rank approximation of the adjacency matrix to determine a short list of candidate nodes. Then Gauss quadrature is applied to rank the nodes in this list. The use of block Lanczos methods is likely to speed up the latter computations.

8.2. Application to directed graphs. This subsection presents computations that illustrate the performance of block Gauss and anti-Gauss quadrature rules associated with the nonsymmetric block Lanczos algorithm. We applied these quadrature rules to eight directed unweighted networks coming from the following 

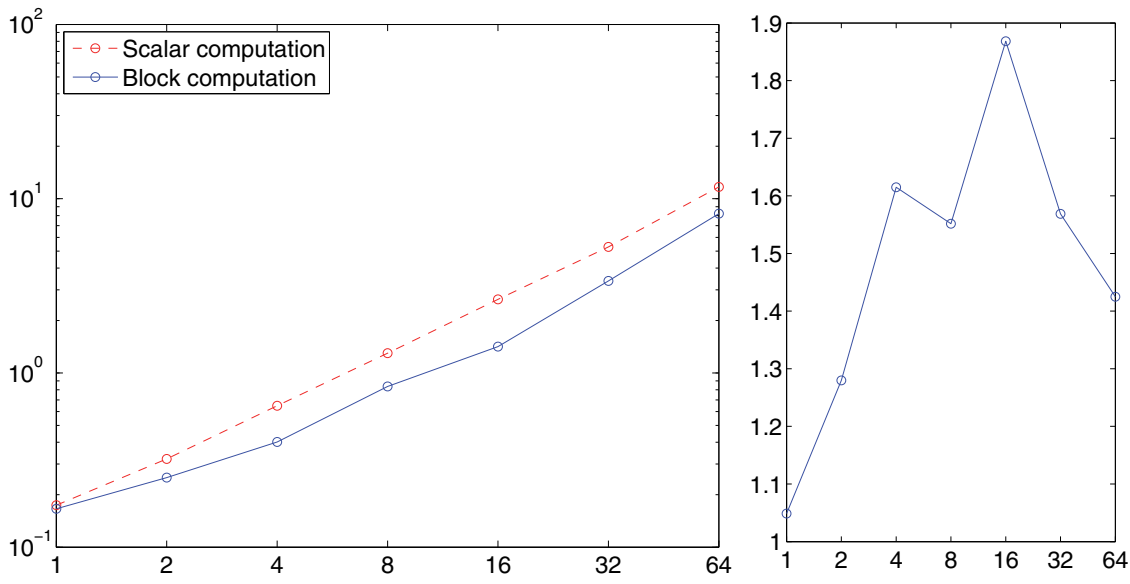

FIG. 8.2. Execution times for block-size 1 (top graph) and block-size $k$ (bottom graph) as a function of $k$ when computing the subgraph centralities of $k$ nodes. The right panel shows the ratio between the timings.

real-world applications:

Airlines (235 nodes, 2101 edges) is a representation of air traffic, available at [27]. The nodes are airports and the directed edges are flights between them.

Celegans (306 nodes, 2345 edges) is the metabolic network of Caenorhabditis elegans [17], a small nematode (roundworm). The data set is available at [1].

Air500 (500 nodes, 24009 edges) is the network of flight connections for the top 500 airports, based on total passenger volume, worldwide [9]. The existence of flight connections between airports is based on flights within one year from July 1, 2007, to June 30, 2008 [39].

Twitter (3556 nodes, 188712 edges) is part of the Twitter network [27]. The nodes are users and the directed edges are mentions and retweets between them.

T2 (9801 nodes, 87025 edges) describes a mesh for a nonlinear diffusion problem, taken from the University of Florida Sparse Matrix Collection [47].

Wikipedia (49728 nodes, 941425 edges) is the structure of Italian Wikipedia. In this graph the nodes are plain articles and the links represent references to other articles. It can be downloaded from [41].

Poisson (85623 nodes, 2374949 edges) is a sparse matrix describing a problem in computational fluid dynamics from the University of Florida Sparse Matrix Collection [47].

Vfem (93476 nodes, 1434636 edges) is a vector finite element complex matrix from a problem in electromagnetics [47]. When computing with this matrix, transposition is replaced by transposition and complex conjugation. Inner products also require complex conjugation.

Thus, all matrices except for T2, Poisson, and Vfem are adjacency matrices. When applying the nonsymmetric block Lanczos algorithm to an arbitrary dense matrix breakdown rarely occurs. However, when $A$ is a general large and sparse nonsymmetric adjacency matrix and the initial vectors are axis vectors $\boldsymbol{e}_{i}$, and, therefore, very sparse, chances of breakdown are high. For instance, if the matrix $A$ only has a few nonzero entries in each row and column, even though the matrices $S_{1}$ and $R_{1}$ in (4.4) are likely to be of full rank, they almost surely will satisfy $S_{1}^{T} R_{1}=O_{k}$, resulting in serious breakdown at the first step. The reason for this difficulty is that independent high- 
dimensional vectors with only a few nonzero entries are likely to be orthogonal. We remark that this problem does not occur with the symmetric block Lanczos method, because this method requires only the matrix $R_{1}$ to be of full rank. The probability of breakdown during the first steps of the nonsymmetric Lanczos method decreases when introducing an additional dense starting vector. This is shown by Bai, Day, and Ye [2]; see below for illustrations.

Suppose that we would like to compute all communicabilities between nodes 1 through $k-1$ and their centralities for a total of $(k-1)^{2}$ numerical quantities. If we try to approximate $\mathcal{I} f=W^{T} f(A) V$ with $W=V=\left[\boldsymbol{e}_{1}, \ldots, \boldsymbol{e}_{k-1}\right]$ using the nonsymmetric block Lanczos method with block-size $k-1$, then breakdown is likely to occur at an early stage of the computations. To reduce the likelihood of breakdown, we append the vector $\boldsymbol{c}$ with all entries one to $W$ and $V$. Thus, we use $W=V=$ $\left[\boldsymbol{e}_{1}, \ldots, \boldsymbol{e}_{k-1}, \boldsymbol{c}\right]$. Then the computations also provide bounds for the starting and ending conveniences of the first $k-1$ nodes provided that an expansion of the integrand in terms of biorthogonal polynomials converges sufficiently rapidly; see the comment at the end of subsection 6.2. The desired $f$-communicabilities are the entries of the leading principal $(k-1) \times(k-1)$ submatrix of the $k \times k$ matrix $\mathcal{I} f=W^{T} f(A) V$. We remark that the block Lanczos method is applied to an orthonormalization of the columns in $V$ and $W$; see below.

Now assume that we would like to compute certain $f$-communicabilities with a small absolute error $\tau$. Using the quantities $T_{N}, F_{N}$, and $G_{N}$ in (8.2)-(8.3), a natural stopping criterion is provided by $T_{N}<\tau$. We show the performance of block Gauss and anti-Gauss quadrature rules when determining approximations of the entries of $\mathcal{I} f=W^{T} \exp (A) V$ with $W=V=\left[\boldsymbol{e}_{1}, \ldots, \boldsymbol{e}_{5}, \boldsymbol{c}\right]$ and $\tau=10^{-3}$.

The initial blocks have to satisfy $V^{T} W=I_{k}$. To accomplish this, in principle, we could orthogonalize the columns of the matrix $W$ defined above by a QR factorization, but this would not necessarily change its sparsity pattern and, therefore, would likely lead to breakdown of the nonsymmetric block Lanczos method. In fact, when $A$ and $W$ are sparse, either one of matrices $R_{1}$ and $S_{1}$ in (4.4), or both, may be singular. For this reason, we redefine the blocks $W$ and $V$ with the aid of the singular value decomposition of the $k \times k$ block $W^{T} V$. If $W^{T} V=U \Sigma Z^{T}$, then the matrices

$$
W_{1}=W U \Sigma^{-1 / 2}, \quad V_{1}=V Z \Sigma^{-1 / 2}
$$

satisfy $W_{1}^{T} V_{1}=I_{k}$ and have dense columns. The sought quantities can be determined from

$$
W^{T} f(A) V=U \Sigma^{1 / 2}\left(W_{1}^{T} f(A) V_{1}\right) \Sigma^{1 / 2} Z^{T} .
$$

To begin with, we repeated the first experiment of section 8.1. Let the matrix $W$ be defined as described above. We computed approximations of $\left[W^{T} f(A) W\right]_{i j}$ by pairs of $N$-block Gauss and $(N+1)$-block anti-Gauss rules, which were computed with the nonsymmetric block Lanczos method, and compared the 36 entries of the resulting matrices to the output of expm. This test was performed on the first four networks, whose size allows the application of expm. The inequalities (8.4) held in all but a small number of cases: One of the inequalities was violated for 5 (out of 36) entries for the Celegans network and 14 of the entries for the Twitter network. The required accuracy was attained for all examples, that is, the error $G_{N}$ (8.3) was smaller than the stopping tolerance $\tau$ for all networks.

Table 8.3 shows the execution times (in seconds) of the MATLAB function expm and the nonsymmetric block Lanczos method. The table also reports the number 
of matrix-vector product evaluations, the number of block Lanczos steps, and the quantity (8.3). Very few block Lanczos steps are needed to determine the desired quantities with required accuracy. For the larger networks, we are unable to evaluate the function expm and, therefore, the error $G_{N}$.

TABLE 8.3

Execution times (in seconds) of expm and the nonsymmetric block Lanczos method. The table also shows the number of matrix-vector product evaluations, the number of block Lanczos steps, and the quantity (8.3).

\begin{tabular}{c|c|c|c|cccc}
\hline & & & expm & \multicolumn{4}{|c}{ Block Lanczos method } \\
Matrix & Nodes & Edges & time & Time & MVP & Steps & $G_{N}$ \\
\hline Airlines & 235 & 2101 & $2.4 \mathrm{e}-01$ & $5.7 \mathrm{e}-02$ & 66 & 6 & $2.8 \mathrm{e}-08$ \\
Celegans & 306 & 2345 & $1.6 \mathrm{e}-01$ & $1.7 \mathrm{e}-02$ & 66 & 6 & $8.5 \mathrm{e}-05$ \\
Air500 & 500 & 24009 & $9.8 \mathrm{e}-02$ & $3.0 \mathrm{e}-02$ & 66 & 6 & $3.1 \mathrm{e}-08$ \\
Twitter & 3656 & 188712 & $4.4 \mathrm{e}+02$ & $7.6 \mathrm{e}-02$ & 90 & 8 & $4.1 \mathrm{e}-13$ \\
T2 & 9801 & 87025 & - & $7.4 \mathrm{e}-02$ & 42 & 4 & - \\
Wikipedia & 49728 & 941425 & - & $7.8 \mathrm{e}-01$ & 90 & 8 & - \\
Poisson & 85623 & 2374949 & - & $6.3 \mathrm{e}-01$ & 30 & 3 & - \\
Vfem & 93476 & 1434636 & - & $6.6 \mathrm{e}-01$ & 18 & 2 & - \\
\hline
\end{tabular}

9. Conclusion. We derived block anti-Gauss quadrature rules and showed how they can be computed by the symmetric or nonsymmetric block Lanczos methods. Application of these rules to the determination of upper and lower bounds, or estimates thereof, for the entries of expressions of the form (1.1) and (1.2) were described. Computed examples that apply quadrature rules to network analysis were presented. We approximated quantities defined by Estrada and collaborators, as well as a few new ones proposed in this paper. The numerical examples illustrate that block methods can be cheaper to apply than a sequence of standard Gauss-type rules (for block-size one), and much cheaper than the evaluation of the matrix exponential.

Acknowledgments. The authors would like to thank the referees for comments. LR would like to thank Giuseppe Rodriguez and Sebastiano Seatzu for an enjoyable visit to Cagliari.

\section{REFERENCES}

[1] Alex Arena's data sets, http://deim.urv.cat/ aarenas/data/welcome.htm.

[2] Z. BAI, D. DAY, AND Q. YE, ABLE: An adaptive block Lanczos method for non-Hermitian eigenvalue problems, SIAM J. Matrix Anal. Appl., 20 (1999), pp. 1060-1082.

[3] Z. Bai, M. Fahey, And G. Golub, Some large-scale matrix computation problems, J. Comput. Appl. Math., 74 (1996), pp. 71-89.

[4] V. BatagelJ and A. Mrvar, Pajek data sets, 2006; available online at http://vlado.fmf.unilj.si/pub/networks/data/.

[5] M. Benzi AND P. Boito, Quadrature rule-based bounds for functions of adjacency matrices, Linear Algebra Appl., 433 (2010), pp. 637-652.

[6] M. Benzi, E. Estrada, AND C. KLymko, Ranking hubs and authorities using matrix functions, Linear Algebra Appl., 438 (2013), pp. 2447-2474.

[7] M. Benzi And G. H. Golub, Bounds for the entries of matrix functions with applications to preconditioning, BIT, 39 (1999), pp. 417-438.

[8] D. A. Bini, G. M. Del Corso, and F. Romani, Evaluating scientific products by means of citation-based models: A first analysis and validation, Electron. Trans. Numer. Anal., 33 (2008), pp. 1-16.

[9] Biological Networks Data Sets of Newcastle University, http://www.biological-networks.org/. 
[10] F. Bonchi, P. Esfandiar, D. F. Gleich, C. Greif, and L. V. S. Lakshmanan, Fast matrix computations for pairwise and columnwise commute times and Katz scores, Internet Math., 8 (2012), pp. 73-112.

11] A. Bounamidi, K. Jbilou, L. Reichel, and H. Sadok, A generalized global Arnoldi method for ill-posed matrix equations, J. Comput. Appl. Math., 236 (2012), pp. 2078-2089.

[12] C. Brezinski and M. Redivo Zaglia, Rational extrapolation for the PageRank vector, Math. Comp., 77 (2008), pp. 1585-1598.

[13] C. Brezinski, M. Redivo Zaglia, And H. SAdok, New look-ahead Lanczos-type algorithms for linear systems, Numer. Math., 83 (1999), pp. 53-85.

[14] D. Calvetti, P. C. Hansen, and L. Reichel, L-curve curvature bounds via Lanczos bidiagonalization, Electron. Trans. Numer. Anal., 14 (2002), pp. 20-35.

[15] D. Calvetti, L. Reichel, And F. Sgallari, Application of anti-Gauss quadrature rules in linear algebra, in Applications and Computation of Orthogonal Polynomials, W. Gautschi, G. H. Golub, and G. Opfer, eds., Birkhäuser, Basel, 1999, pp. 41-56.

[16] J. J. Croft, E. Estrada, D. J. Higham, and A. Taylor, Mapping directed networks, Electron. Trans. Numer. Anal., 37 (2010), pp. 337-350.

[17] J. Duch And A. Arenas, Community identification using extremal optimization, Phys. Rev. E, $72(2005), 027104$.

[18] A. J. Duran and P. Lopez-Rodriguez, Orthogonal matrix polynomials: Zeros and Blumenthal's theorem, J. Approx. Theory, 84 (1996), pp. 96-118.

[19] E. Estrada and N. Hatano, Communicability in complex networks, Phys. Rev. E, 77 (2008), 036111.

[20] E. Estrada, N. Hatano, And M. Benzi, The physics of communicability in complex networks, Phys. Rep., 514 (2012), pp. 89-119.

[21] E. Estrada And D. J. Higham, Network properties revealed through matrix functions, SIAM Rev., 52 (2010), pp. 696-714.

[22] E. Estrada, D. J. Higham, and N. Hatano, Communicability betweenness in complex networks, Phys. A, 388 (2009), pp. 764-774.

[23] E. Estrada and J. A. RodríGuez-Veláquez, Subgraph centrality in complex networks, Phys. Rev. E, 71 (2005), 056103.

[24] E. Estrada And J. A. RodríGuez-Veláquez, Subgraph centrality and clustering in complex hyper-networks, Phys. A, 364 (2006), pp. 581-594.

[25] C. Fenu, D. Martin, L. Reichel, and G. Rodriguez, Network analysis via partial spectral factorization and Gauss quadrature, SIAM J. Sci. Comput., 35 (2013), pp. A2046-A2068.

[26] K. Gallivan, M. Heath, E. Ng, B. Peyton, R. Plemmons, J. Ortega, C. Romine, A. Samen, And R. Voigt, Parallel Algorithms for Matrix Computations, SIAM, Philadelphia, 1990.

[27] Gephi Sample Data Sets, http://wiki.gephi.org/index.php/Datasets.

[28] G. H. Golub and G. Meurant, Matrices, moments and quadrature, in Numerical Analysis 1993, D. F. Griffiths and G. A. Watson, eds., Longman, Harlow, UK, 1994, pp. 105-156.

[29] G. H. Golub and G. Meurant, Matrices, Moments and Quadrature with Applications, Princeton University Press, Princeton, 2010.

[30] G. H. Golub AND U. von Matt, Quadratically constrained least squares and quadratic problems, Numer. Math., 59 (1991), pp. 561-580.

[31] G. H. Golub and U. von Matt, Generalized cross-validation for large-scale problems, J. Comput. Graph. Statist., 6 (1997), pp. 1-34.

[32] R. Guimerì, L. Danon, A. Díaz-Guilera, F. Giralt, and A. Arenas, Self-similar community structure in a network of human interactions, Phys. Rev. E, 68 (2003), 065103(R).

[33] V. E. Henson And G. SAnders, Locally supported eigenvectors and matrices associated with connected and unweighted power-law graphs, Electron. Trans. Numer. Anal., 39 (2012), pp. 353-378.

[34] N. J. Higham, Functions of Matrices: Theory and Computation, SIAM, Philadelphia, 2008.

[35] H. Jeong, S. Mason, A.-L. Barabási, And Z. N. Oltvai, Lethality and centrality of protein networks, Nature, 411 (2001), pp. 41-42.

[36] J. V. LAmbers, Enhancement of Krylov subspace spectral methods by block Lanczos iteration, Electron. Trans. Numer. Anal., 31 (2008), pp. 86-109.

[37] D. P. Laurie, Anti-Gaussian quadrature formulas, Math. Comp., 65 (1996), pp. 739-747.

[38] G. López Lagomasino, L. Reichel, and L. Wunderlich, Matrices, moments, and rational quadrature, Linear Algebra Appl., 429 (2008), pp. 2540-2554.

[39] J. Marcelino And M. Kaiser, Critical paths in a metapopulation model of H1N1: Efficiently delaying influenza spreading through flight cancellation, PLoS Currents Influenza, arXiv:1205.3245, 2012. 
[40] The Max Plank Institute for Software Systems web site, http://socialnetworks.mpi-sws.org/ data-wosn2009.html.

[41] Lev Muchnik's Data Sets web page, http://www.levmuchnik.net/Content/Networks/ NetworkData.html.

[42] M. E. J. Newman, The structure of scientific collaboration networks, Proc. Natl. Acad. Sci. USA, 98 (2001), pp. 404-409.

[43] Mark Newman's web page, http://www-personal.umich.edu/ mejn/netdata/.

[44] L. Reichel, G. Rodriguez, and S. Seatzu, Error estimates for large-scale ill-posed problems, Numer. Algorithms, 51 (2009), pp. 341-361.

[45] A. Sinap And W. VAn Assche, Polynomial interpolation and Gaussian quadrature for matrixvalued functions, Linear Algebra Appl., 207 (1994), pp. 71-114.

[46] S. Sun, L. Ling, N. Zhang, G. Li, And R. Chen, Topological structure analysis of the proteinprotein interaction network in budding yeast, Nucleic Acids Research, 31 (2003), pp. 24432450.

[47] The University of Florida Sparse Matrix Collection, http://www.cise.ufl.edu/research/sparse/ matrices/.

[48] B. Viswanath, A. Mislove, M. Cha, And K. P. Gummadi, On the evolution of user interaction in Facebook, in Proceedings of the 2nd ACM SIGCOMM Workshop on Social Networks (WOSN'09), Barcelona, Spain, 2009, pp. 37-42.

[49] D. J. Watts and S. H. Strogatz, Collective dynamics of 'small-world' networks, Nature, 393 (1998), pp. 440-442. 